added this macro

LAVAL-PHY-99-20

\title{
Generating-function method for fusion rules
}

\author{
L. Bégin吧, C. Cummins ${ }^{\sharp 2}$ and P. Mathieu明 \\ घ Département de Physique, Université Laval, Québec, Canada G1K 7P4 \\ \# Mathematics Department, University of Concordia, Montréal Québec Canada H3G 1 M8
}

\begin{abstract}
This is the second of two articles devoted to an exposition of the generating-function method for computing fusion rules in affine Lie algebras. The present paper focuses on fusion rules, using the machinery developed for tensor products in the companion article. Although the Kac-Walton algorithm provides a method for constructing a fusion generating function from the corresponding tensor-product generating function, we describe a more powerful approach which starts by first defining the set of fusion elementary couplings from a natural extension of the set of tensor-product elementary couplings. A set of inequalities involving the level are derived from this set using Farkas' lemma. These inequalities, taken in conjunction with the inequalities defining the tensor products, define what we call the fusion basis. Given this basis, the machinery of our previous paper may be applied to construct the fusion generating function. New generating functions for $\widehat{s p}(4)$ and $\widehat{s u}(4)$, together with a closed form expression for their threshold levels are presented.
\end{abstract}

05/99, revised 04/00 (arXiv:hepth/0005002)

1 Work supported by NSERC (Canada).

2 Work supported by NSERC (Canada) and FCAR (Québec). 


\section{Introduction}

The basic definition of a fusion coefficient is that it gives the number of independent couplings between three different fields in conformal field theory (cf. also the introduction of [1]; for a review of conformal field theory, and in particular fusion rules, see [2]). Even in theories with a Lie group symmetry, the so-called Wess-Zumino-Witten (WZW) models, an intrinsic conformal-field theoretical characterisation is unavoidable. This is manifest in formulae for the fusion coefficients: the most fundamental one is the Verlinde formula [3], that expresses a fusion coefficient in terms of modular $S$ matrix elements:

$$
\mathcal{N}_{\hat{\lambda} \hat{\mu}}^{(k) \hat{\nu}}=\sum_{\hat{\sigma} \in P_{+}^{k}} \frac{S_{\hat{\lambda} \hat{\sigma}} S_{\hat{\mu} \hat{\sigma}} S_{\hat{\nu} \hat{\sigma}}^{*}}{S_{0 \hat{\sigma}}}
$$

Here we use notation appropriate to a WZW model in which primary fields are in one-toone correspondence with the integrable representations of the spectrum-generating affine algebra at a fixed level $k$ (this set is denoted by $P_{+}^{k}$ ) and 0 stands for the basic representation, whose finite projection is the scalar representation. Fields are not distinguished from their representation labels. The matrix $S$ specifies the linear modular transformation properties of the characters of the primary fields among themselves. Up to a constant fixed by unitarity, it takes the form

$$
S_{\hat{\lambda} \hat{\mu}} \sim \sum_{w \in W} \epsilon(w) \exp \left(-\frac{2 \pi i}{k+g}(w(\lambda+\rho), \mu+\rho)\right)
$$

where $g$ stands for the dual Coxeter number of the algebra under consideration, $\rho$ is the Weyl vector, $\lambda$ is the finite projection of the affine weight $\hat{\lambda}$ and $W$ is the finite Weyl group.

The remarkable fact that the ratio of two $S$ matrix elements is a finite character evaluated at a special point yields a close relation between fusion and tensor-product coefficients. Indeed, since the finite character and its evaluation read

$$
\chi_{\lambda}=\frac{\sum_{w \in W} \epsilon(w) e^{w(\lambda+\rho)}}{\sum_{w \in W} \epsilon(w) e^{w \rho}} \quad \text { and } \quad \chi_{\lambda}(\xi)=\frac{\sum_{w \in W} \epsilon(w) e^{(w(\lambda+\rho), \xi)}}{\sum_{w \in W} \epsilon(w) e^{(w \rho, \xi)}}
$$

we observe that

$$
\chi_{\lambda}(\xi)=\frac{S_{\hat{\lambda}, \hat{\sigma}}}{S_{0, \hat{\sigma}}} \quad \text { with } \quad \xi=-\frac{2 \pi i}{k+g}(\sigma+\rho)
$$

This leads to the Kac-Walton formula which relates the fusion and the tensor-product coefficients. 
The Verlinde formula does not make manifest the basic integrality property of the fusion coefficients. The $S$ matrix elements being in general complex numbers, it is not even clear at first sight that the fusion coefficients are real (this follows from the unitarity property of $S$ ). The integrality is ensured by the Kac-Walton formula, but in this case the positivity is not manifest.

It is mainly with the aim of displaying manifestly non-negative formulae for fusion rules that we have looked for fusion generating functions [4]. Although the construction of explicit generating functions has an intrinsic interest, we regard the unravelling of the concept of threshold level - reviewed below - as being the most important outcome of this analysis. It leads to a complete characterisation of fusion coefficients in terms of the corresponding tensor-product coefficients and a set of threshold levels.

As a result, the interest has shifted from the construction of fusion generating functions to the search for threshold-level computing techniques. For $\widehat{s u}(N), N=2,3$, 4 , it has been found that the threshold level is coded in a simple way in the Berenstein-Zelevinsky triangles [5] (cf. also section 7.1 of [1]) describing the various distinct couplings of a tensor product $[6,7]$. However, these formulae are difficult to generalise to larger values of $N$. Moreover, this approach, based on a diagrammatic description of the tensor product, is limited to the $\widehat{s u}(N)$ algebras.

The aim of the present paper is to apply the machinery developed in [1] to these problems. We find new generating functions for $\widehat{s p}(4)$ and $\widehat{s u}(4)$, together with a closed form expression for their threshold levels. More importantly, we introduce the concept of fusion basis, that is, the set of linear and homogeneous Diophantine inequalities that describes completely the fusion rules.

The article is organised as follows. In section 2, after introducing some notation, we present a brief review of fusion rules and show, with the example of $\widehat{s u}(2)$, how tensorproduct generating functions and the Kac-Walton algorithm can be used to construct fusion-rule generating functions. A more powerful approach to the problem is then elaborated in section 3. It relies on the conjectural existence of a linear and homogeneous set of inequalities that provides a complete description of fusion rules. Given a set of fusion elementary couplings, Farkas' lemma is then used as a technique to extract the underlying inequalities. This is what we call a fusion basis, i.e., the basis in terms of which these fusion elementary couplings are the elementary solutions. A complete analysis of the $\widehat{s u}(3), \widehat{s p}(4)$ 
and $\widehat{s u}(4)$ cases is presented in section 4,5 and 6 respectively. In all three cases, the general expression for the threshold levels is obtained explicitly. Various arguments (based on Giambelli-type formula and level-rank duality) supporting our results are presented in Appendix A. In Appendix B, we recall previous conjectures and clarify their relation to those formulated here.

\section{Fusion rules}

Let $\widehat{g}$ be the affine Lie algebra corresponding to the finite Lie algebra $g$. Quantities with hats generally refers to $\widehat{g}$. The fundamental weights of $\widehat{g}$ are denoted by $\widehat{\omega}_{i}, i=0,1, \ldots, r$, where $r$ is the rank of $g$. An affine weight may be written as

$$
\hat{\lambda}=\sum_{i=0}^{r} \lambda_{i} \widehat{\omega}_{i}=\left[\lambda_{0}, \lambda_{1}, \ldots, \lambda_{r}\right]
$$

If the Dynkin labels $\lambda_{i}$ are nonnegative, the weight $\hat{\lambda}$ is the highest weight of an integrable representation of $\widehat{g}$ at level $k$, with $k$ defined by

$$
k=\sum_{i=0}^{r} \lambda_{i} a_{i}^{\vee}
$$

The $a_{i}^{\vee}$ are the co-marks: $a_{0}^{\vee}=1$, and the remaining $a_{i}^{\vee}$ are the coefficients of expansion of the longest root of $g$ in terms of the simple coroots. The set of such weights is denoted $P_{+}^{k}$

To the affine weight $\hat{\lambda}$, we associate a weight $\lambda$ of the finite algebra $g$

$$
\lambda=\sum_{i=1}^{r} \lambda_{i} \omega_{i}=\left(\lambda_{1}, \ldots, \lambda_{r}\right)
$$

where $\omega_{i}$ for $(i=1, \ldots, r)$ are the fundamental weights of $g . \hat{\lambda}$ is thus uniquely fixed from $\lambda$ and $k$. The set of integrable finite weights is written $P_{+}$.

In the conformal field-theory context, fusion rules yield the number of independent couplings between three given primary fields. Here we are interested in fusion rules in WZW models $[8,9]$, whose generating spectrum algebra is an affine Lie algebra at integer level. 
Denote the multiplicity of the representation $\hat{\nu}$ in the fusion rule $\hat{\lambda} \times \hat{\mu}$ by

$$
\hat{\lambda} \times \hat{\mu}=\sum_{\hat{\nu} \in P_{+}^{k}} \mathcal{N}_{\hat{\lambda} \hat{\mu}}^{(k) \hat{\nu}} \hat{\nu}
$$

and denote by $\mathcal{N}_{\lambda \mu}{ }^{\nu}$ the multiplicity of the representation $\nu$ in the tensor product $\lambda \otimes \mu$ :

$$
\lambda \otimes \mu=\sum_{\nu \in P_{+}} \mathcal{N}_{\lambda \mu}{ }^{\nu} \nu
$$

where by abuse of notation, we use the same symbol for the highest weight and the highestweight representation. The precise relation between tensor-product and fusion-rule coefficients is given by the Kac-Walton formula $[10,11,12]$ :

$$
\mathcal{N}_{\hat{\lambda} \hat{\mu}}^{(k)^{\hat{\nu}}}=\sum_{\substack{\xi \in P_{+} \\ w \in \widehat{W}, w \cdot \hat{\xi}=\hat{\nu} \in P_{+}^{k}}} \mathcal{N}_{\lambda \mu}^{\xi} \epsilon(w)
$$

$w$ is an element of the affine Weyl group $\widehat{W}$, of $\operatorname{sign} \epsilon(w)$, and the dot indicates the shifted action,

$$
w \cdot \hat{\lambda}=w(\hat{\lambda}+\hat{\rho})-\hat{\rho} \quad \hat{\rho}=\sum_{i=0}^{r} \widehat{\omega}_{i}
$$

The Kac-Walton formula can be transformed into a simple algorithm: one first calculates the tensor product of the corresponding finite weights and then extends every weight to its affine version at the appropriate value of $k$ and shift-reflects back to the integrable affine sector those weights which have negative zeroth Dynkin label. Weights that cannot be shift-reflected in the integrable sector are ignored (for example this is the case for those which have zeroth Dynkin label equal to -1 ).

The affine extension of the weights that occur in the tensor product may not be integrable at level $k$ but are integrable at level $2 k$. If we divide the weight space into domains that are mapped into each other by the application of the affine Weyl reflections, then the affine reflections which contribute to the Kac-Walton algorithm, apart from the identity, are those corresponding to the domains next to the fundamental alcove and which lies in the $P_{+}$cone. This is a crucial property of the Kac-Walton algorithm for its application to the construction of fusion-rule generating functions. Let us denote by $\widehat{W}_{f}$ this finite 
subset of the affine Weyl group that need to be considered . For instance, the elements of $\widehat{W}_{f}$ for the lowest rank algebras are:

$$
\begin{array}{ll}
\widehat{s u}(2): & \widehat{W}_{f}=\left\{i d, s_{0}\right\} \\
\widehat{s u}(3): & \widehat{W}_{f}=\left\{i d, s_{0}, s_{1} s_{0}, s_{2} s_{0}\right\} \\
\widehat{s u}(4): & \widehat{W}_{f}=\left\{i d, s_{0}, s_{1} s_{0}, s_{3} s_{0}, s_{2} s_{1} s_{0}, s_{2} s_{3} s_{0}, s_{1} s_{3} s_{0}, s_{0} s_{1} s_{3} s_{0}\right\} \\
\widehat{s p}(4): & \widehat{W}_{f}=\left\{i d, s_{0}, s_{1} s_{0}, s_{0} s_{1} s_{0}\right\} \\
\widehat{G}_{2}: & \widehat{W}_{f}=\left\{i d, s_{0}, s_{1} s_{0}, s_{2} s_{0}, s_{0} s_{2} s_{1} s_{0}\right\}
\end{array}
$$

where $s_{i}$ denotes the reflection with respect to the root $\alpha_{i}$. This set of elements $w$ can be characterised as follows: these are the elements $w$ of the affine Weyl group that satisfy the requirement:

$$
w\left\{2 \alpha_{0}^{\vee}+\alpha_{1}^{\vee}+\cdots+\alpha_{r}^{\vee}, \alpha_{1}^{\vee}, \cdots, \alpha_{r}^{\vee}\right\} \in \Delta_{+}^{\vee}
$$

where $\Delta_{+}^{\vee}$ stands for the set of positive real coroots of the affine algebra under consideration and $r$ stands for its rank. This condition is adapted from [13] as further analysed in [14].

Note also that (2.6) may be rewritten as:

$$
\hat{\nu} \in P_{+}^{k}: \quad \mathcal{N}_{\hat{\lambda} \hat{\mu}}^{(k)^{\hat{\nu}}}=\sum_{w \in \widehat{W}_{f}^{-1}, w \cdot \hat{\nu} \in P_{+}} \mathcal{N}_{\lambda \mu}{ }^{w \cdot \hat{\nu}} \epsilon(w)
$$

where it is understood that $w \cdot \hat{\nu}$ stands for its finite part since it is an index of the tensorproduct coefficient. This allows us to study in isolation the contribution of a single weight in the fusion. For instance, for $\widehat{s u}(2)$ that reads

$$
\mathcal{N}_{\hat{\lambda} \hat{\mu}}^{(k)^{\hat{\nu}}}=\mathcal{N}_{\lambda \mu}{ }^{\nu}-\mathcal{N}_{\lambda \mu}{ }^{s_{0} \cdot \hat{\nu}}=\mathcal{N}_{\lambda_{1} \mu_{1}}^{\nu_{1}}-\mathcal{N}_{\lambda_{1} \mu_{1}}{ }^{2 k+2-\nu_{1}}
$$

Here is an illustrative example of the Kac-Walton algorithm that will also serve to introduce the key notion of threshold level. Take the following $s p(4)$ tensor product: $(1,1) \otimes(1,1)$. Its decomposition reads

$$
(1,1) \otimes(1,1)=(0,0) \oplus(0,1) \oplus 2(2,0) \oplus(0,2) \oplus(0,3) \oplus 2(2,1) \oplus(2,2) \oplus(4,0)
$$

The $s p(4)$ comarks are all equal to one so that the affine extension of a weight $(m, n)$ at level $k$ is $[k-m-n, m, n]$. At level 2 , the weights $(0,3)$ and $(2,1)$ are ignored (they have $\nu_{0}=-1$ ) and the remaining non-integrable weights are $[-2,2,2]$ and $[-2,4,0]$. Since the zeroth 
simple root is $\hat{\alpha}_{0}=[2,-2,0]$, we have $s_{0} \cdot[-2,2,2]=[0,0,2]$ and $s_{0} \cdot[-2,4,0]=[0,2,0]$, so that the resulting fusion is

$$
[0,1,1] \times[0,1,1]=[2,0,0] \oplus[1,0,1] \oplus[0,2,0]
$$

In the above example, we see that the weights $(0,0),(0,1),(2,0)$ appear first at level 2. It is easily checked that they reappear at every level $k \geq 2$. We then say that their threshold level, usually denoted by $k_{0}$, is 2 . The threshold level is thus the smallest value of $k$ such that the fusion coefficient $\mathcal{N}_{\hat{\lambda} \hat{\mu}}^{(k) \hat{\nu}}$ is non-zero. If we indicate the threshold level by a subindex, by considering the extension of the above tensor product at different levels, we find

$$
\begin{aligned}
&(1,1) \otimes(1,1)=(0,0)_{2} \oplus(0,1)_{2} \oplus(2,0)_{2} \oplus(2,0)_{3} \oplus(0,2)_{3} \\
& \oplus(0,3)_{3} \oplus 2(2,1)_{3} \oplus(2,2)_{4} \oplus(4,0)_{4}
\end{aligned}
$$

To read off a fusion at fixed level $k$, we only keep terms with index not greater than $k$. The concept of threshold level was first introduced in [4]. It can be shown (cf. ref. [6]) that the existence of a threshold level is a consequence the depth rule of Gepner and Witten [9]. The notion of threshold level implies directly that

$$
\mathcal{N}_{\hat{\lambda} \hat{\mu}}^{(k) \hat{\nu}} \leq \mathcal{N}_{\hat{\lambda} \hat{\mu}}^{(k+1) \hat{\nu}} \quad \text { and } \quad \lim _{k \rightarrow \infty} \mathcal{N}_{\hat{\lambda} \hat{\mu}}^{(k) \hat{\nu}}=\mathcal{N}_{\lambda \mu}{ }^{\nu}
$$

To the triplet $(\lambda, \mu, \nu)$ there corresponds $\mathcal{N}_{\lambda \mu}{ }^{\nu}$ distinct couplings, hence $\mathcal{N}_{\lambda \mu}{ }^{\nu}$ values of $k_{0}$, one for each distinct coupling. Let us denote these by $k_{0}^{(i)}, i=1, \ldots, \mathcal{N}_{\lambda \mu}{ }^{\nu}$, implementing in this notation the natural ordering $k_{0}^{(i)} \leq k_{0}^{(i+1)}$. Then

$$
\mathcal{N}_{\hat{\lambda} \hat{\mu}}^{(k) \hat{\nu}}=\left\{\begin{array}{cccc}
\max (i) & \text { if } \quad k \geq k_{0}{ }^{(i)} & \text { and } & \mathcal{N}_{\lambda \mu}{ }^{\nu} \neq 0 \\
0 & \text { if } \quad k<k_{0}{ }^{(1)} & \text { or } & \mathcal{N}_{\lambda \mu}{ }^{\nu}=0
\end{array}\right.
$$

Further variations on the idea of threshold level are presented in [15].

Let us finally note that the fusion coefficients are invariant under the following action of the outer-automorphism group [10]

$$
\mathcal{N}_{A \hat{\lambda}, A^{\prime} \hat{\mu}}^{(k)}{ }^{A A^{\prime} \hat{\nu}}=\mathcal{N}_{\hat{\lambda} \hat{\mu}}^{(k) \hat{\nu}}
$$


For example, for $\widehat{s p}(4)$, the non-trivial outer automorphism $a$ exchanges the zeroth and second root, or equivalently, it acts on weights as $a\left[\lambda_{0}, \lambda_{1}, \lambda_{2}\right]=\left[\lambda_{2}, \lambda_{1}, \lambda_{0}\right]$. Acting on the fusion $(2.13)$ as

$$
a[0,1,1] \times a[0,1,1]=[1,1,0] \times[1,1,0]=[2,0,0] \oplus[1,0,1] \oplus[0,2,0]
$$

which is easily checked from the tensor product

$$
(1,0) \otimes(1,0)=(0,0) \oplus(0,1) \oplus(2,0)
$$

which is non-truncated at level 2. Other fusions at level 2 can be obtained from (2.13) by acting on the weights as follows

$$
\begin{aligned}
a[0,1,1] \times[0,1,1]=[1,1,0] \times[0,1,1] & =a[2,0,0] \oplus a[1,0,1] \oplus a[0,2,0] \\
& =[0,0,2] \oplus[1,0,1] \oplus[0,2,0]
\end{aligned}
$$

The algorithm underlying the Kac-Walton formula suggests a simple road to the construction of fusion-rule generating functions, that is by starting from the tensor-product calculation, but keeping track of the level and taking into account the action of the affine Weyl group. We illustrate the method for the simple $\widehat{s u}(2)$ case. Recall that the $s u(2)$ tensor-product generating function reads

$$
G^{s u(2)}(L, M, N)=\frac{1}{(1-L M)(1-L N)(1-M N)}
$$

We start with the generating function

$$
F(d, L, M, N)=\frac{1}{(1-d)(1-L M)(1-L N)(1-M N)} .
$$

This is just the generating function for $s u(2)$ tensor products divided by $(1-d)$. The exponent of $d$ will be identified with the level. We will proceed to the generating function for $\widehat{s u}(2)$ fusion rules by modifying (2.22). First note that at level $k$ we need only consider the products of $s u(2)$ representations $(a)$ with $a \leq k$. The generating function (2.22) includes products of representations which violate this condition. To keep terms of the form $d^{k} L^{a}$ with $a \leq k$ introduce a dummy variable $x$ (using MacMahon's notation - cf. [1])

$$
\stackrel{x}{\Omega} \frac{1}{\left(1-x^{-1}\right)} F\left(d x, L x^{-1}, M, N\right)
$$


This first converts $d^{k} L^{a}$ to $x^{-m+k-a} d^{k} L^{a}$, with $m \geq 0$ and then keeps terms of degree zero in $x$ which corresponds to keeping the terms of $F(d, L, M, N)$ with $a \leq k$ as required. This yields:

$$
\frac{1}{(1-d)(1-d L M)(1-d L N)(1-M N)} .
$$

Repeating this procedure with $L$ replaced by $M$ yields:

$$
G(d, L, M, N)=\frac{1-d^{2} L M N^{2}}{(1-d)(1-d L M)(1-d L N)(1-d M N)\left(1-d L M N^{2}\right)} .
$$

This is still a generating function for tensor products, but with the size of the representation Dynkin labels restricted to be less than or equal to the level.

To take into account the affine Weyl group, consider a term in the expansion of the generating function which contains $d^{k} N^{c}$. If $c \geq k+1$ then this representation is reflected back into the fundamental region of the affine Weyl group: $c \mapsto c-2(c-k-1)=-c+2 k+2$ or $d^{k} N^{c} \mapsto d^{k} N^{2 k-c+2}$. Since this is a reflection, the corresponding character must be subtracted. In principle other affine Weyl transformations might be necessary to obtain a weight in the fundamental domain, but, as discussed earlier, for $\widehat{s u}(2)$ one reflection suffices. At the level of generating functions the effect is to replace

$$
G(d, L, M, N) \quad \mapsto \quad G(d, L, M, N)-N^{2} G\left(d N^{2}, L, M, N^{-1}\right)
$$

Note that the new generating function contains terms with negative powers of $N$ and also terms with $c>k$. To obtain the final function we projected out the required terms as above. Although this calculation is somewhat long (the verification here was done on a computer), the final result is very simple:

$$
G^{\widehat{s u}(2)}=\frac{1}{(1-d)(1-d L M)(1-d L N)(1-d M N)}
$$

This has first been written down in [4]. There are thus four elementary couplings:

$$
\begin{array}{lll}
\widehat{E}_{0}: d: & (0) \otimes(0) \supset(0)_{1}, & \widehat{E}_{2}: d L N:(1) \otimes(0) \supset(1)_{1}, \\
\widehat{E}_{1}: d L M:(1) \otimes(1) \supset(0)_{1}, & & \widehat{E}_{3}: d M N:(0) \otimes(1) \supset(1)_{1} .
\end{array}
$$

As explained above, subscripts indicate the threshold level.

The notion of a model was discussed in [1]. A model for this generating function is $\mathbb{Q}\left[\hat{E}_{0}, \hat{E}_{1}, \hat{E}_{2}, \hat{E}_{3}\right]$ with the gradings of $\hat{E}_{0}, \cdots, \hat{E}_{3}$ respectively given by $(1,0,0,0),(1,1,1,0),(1,1,0,1)$ 
and $(1,0,1,1)$ for the ordering $X_{0}=d, X_{1}=L, X_{2}=M, X_{3}=N$. As for the finite $\operatorname{su}(2)$ case, there are no relations between the elementary couplings.

The generalisation of the above calculation to other affine Lie algebras is straightforward. Starting from the tensor-product generating function augmented by the factor $1 /(1-d)$, where $d$ keeps track of the level, one first enforces the integrability requirement of the first two weights (those that are fused together); one then implements all the affine reflections of the set $\widehat{W}_{f}$ on the third weight and projects the alternating sum onto the integrable sector. However, even though the strategy is clear, the computations become rapidly very complicated.

To bypass this difficulty, we have argued (cf. [1] section 3) that the use of a direct description of tensor products in terms of a system of inequalities (e.g., the LittlewoodRichardson (LR) inequalities underlying their combinatorial description for calculating tensor products - cf. [1] section 4) simplifies the general procedure to a very large extent in addition to allowing us to use powerful algebraic results. We now look for a similar procedure here. However, this program faces an immediate difficulty since even for $s u(N)$, a combinatorial description of fusion rules is not known. Our method is instead to find an independent route leading to the elementary couplings. Indeed, the elementary couplings are really what we need in order to apply our Grobner basis machinery. Quite remarkably, it turns out that once elementary couplings are found, there is a method that allows us to reconstruct the underlying system of Diophantine inequalities.

\section{Fusion-rule elementary couplings}

The construction of this section depends upon the following:

Fundamental conjecture: There exists a fusion basis, that is, a set linear and homogeneous inequalities involving $k$ and containing as a subset, a tensor-product basis.

For instance, the LR basis is a set linear and homogeneous inequalities. Every solution can be expanded in terms of the elementary solutions of these inequalities. For $\widehat{s u}(N)$, the conjecture amounts to the existence of a set of additional inequalities involving the level $k$ that provide the proper truncation describing the fusion rules. The relation of this conjecture to the conjectures presented in [4] is discussed in the Appendix B. 
Note that homogeneity is the key property which allows us to reconstruct the fusion basis from a set of fusion elementary couplings using Farkas' Lemma. This condition does not necessarily hold, for example we have found that the Lie superalgebra $\operatorname{csp}(1,2)$ does not have a homogeneous basis.

Given homogeneity and Farkas' lemma, the problem is reduced to finding a set of fusion elementary couplings. The Kac-Walton algorithm is one possible approach, but a rather difficult one. Instead, we will introduce a simpler approach based on the outerautomorphism group. Unfortunately, it relies on another conjecture.

Let us start from the set of tensor-product elementary couplings $\left\{E_{i}, i \in I\right\}$ for some set $I$ fixed by the algebra under study. For each $E_{i}$, we calculate the threshold level $k_{0}\left(E_{i}\right)$. This information specifies the affine extension of $E_{i}$. The affine extension of a tensor-product elementary coupling is necessarily a fusion-rule elementary coupling given our hypothesis that the fusion basis contains, as a subsystem, the set of inequalities that describe tensor products. Denoting by a hat the affine extension of a tensor-product elementary coupling

$$
\widehat{E}_{i}=d^{k_{0}\left(E_{i}\right)} E_{i}
$$

we have then a partial set of fusion elementary couplings with the set $\left\{\widehat{E}_{i}, i \in I\right\}$. Our conjecture is that the missing fusion elementary couplings can all be generated by the action of the outer-automorphism group whenever this group is nontrivial:

The outer-automorphism completeness conjecture: The complete set of elementary couplings $\left\{\widehat{E}_{i}, i \in J\right\}$ for a set $J \supset I$ can be generated by the action of the outer-automorphism group on the set $\left\{\widehat{E}_{i}, i \in I\right\}$, i.e., the full set is contained in $\left\{\mathcal{A} \widehat{E}_{i}\right\}$ :

$$
\left\{\widehat{E}_{i}, i \in J\right\} \subset\left\{\mathcal{A} \widehat{E}_{i}, i \in I\right\}
$$

The action of the outer-automorphism group on a coupling is defined as follows. Let the three weights in the coupling be $\{\hat{\lambda}, \hat{\mu} ; \hat{\nu}\}$ where $\hat{\nu} \subset \hat{\lambda} \times \hat{\mu}$, then

$$
\mathcal{A}\{\hat{\lambda}, \hat{\mu} ; \hat{\nu}\}=\left\{A \hat{\lambda}, A^{\prime} \hat{\mu} ; A A^{\prime} \hat{\nu}\right\}
$$

where $A, A^{\prime}$ are arbitrary elements of the outer-automorphism group; the conjectured completeness requires the consideration of all possible pairs $\left(A, A^{\prime}\right)$. 
It should be stressed that we do not suppose that the action of $\mathcal{A}$ on an elementary coupling will necessarily produce another elementary coupling. Indeed, the resulting coupling could be a product of elementary couplings. What is conjectured here is that all fusion elementary couplings can be generated in this way.

If the outer-automorphism group is trivial, we expect that there will a single extra elementary coupling, the one associated to the scalar coupling: $\widehat{E}_{0}$.

As a simple example consider $\widehat{s u}(2)$. Start with the elementary coupling $E_{1}:(1) \otimes$ (1) $\supset(0)$. It is easy to show that this coupling arises at level 1 . This is thus the value of its threshold level. The corresponding fusion is $[0,1] \times[0,1] \supset[1,0]$. We now consider all possible actions of the outer-automorphims group on it. Since this group is of order 2, there are 4 possible choices for the pair

$$
\left(A, A^{\prime}\right) \in\{(a, a),(1,1),(1, a),(a, 1)\}
$$

with $a\left[\lambda_{0}, \lambda_{1}\right]=\left[\lambda_{1}, \lambda_{0}\right]$. This generates the following set of four elementary couplings found previously (cf. eq (2.28)):

$$
\begin{array}{lll}
\widehat{E}_{0}: d: \quad[1,0] \times[1,0] \supset[1,0] & & \widehat{E}_{2}: d L N:[0,1] \times[1,0] \supset[0,1] \\
\widehat{E}_{1}: d L M:[0,1] \times[0,1] \supset[1,0] & & \widehat{E}_{3}: d M N:[1,0] \times[0,1] \supset[0,1] .
\end{array}
$$

Let us then suppose that we have a complete set of fusion elementary couplings which are the elementary solutions of set of linear and homogeneous inequalities that we are looking for. A standard theorem in the theory of linear Diophantine equations (cf. [16]) states that every non-negative integer solution of a given set of homogeneous Diophantine inequalities for the variables $x_{i}$ (e.g., for $s u(N)$, these are the $\left\{\lambda_{i}, n_{i j}\right\}$ ) can be generated from a non-negative combination of the fundamental solutions. Hence, given the set of elementary couplings $\left\{\widehat{E}_{i}\right\}$, any coupling can be decomposed (maybe not uniquely) in the form $\prod_{i} \widehat{E}_{i}^{a_{i}}$. Let the grading variables representing the $x_{i}$ be denoted by $X_{i}$. To the expression of $g\left(\widehat{E}_{i}\right)$ corresponds a vector $\epsilon_{i}$ of components $\epsilon_{i j}$, which is the vector form of the elementary solutions of the Diophantine equations. In other words,

$$
\widehat{E}_{i}: g\left(\widehat{E}_{i}\right)=\prod_{j} X_{j}^{\epsilon_{i j}}
$$


Reading off a particular coupling means that we are interested in a specific set of nonnegative integers $\left\{x_{i}\right\}$ given by

$$
\sum_{i} a_{i} \epsilon_{i j}=x_{j}
$$

in terms of non-negative integers $a_{i}$. We are thus looking for the existence conditions for such a coupling. This is related to Farkas' lemma $[17,18]$. The standard, rational, form of the lemma is (cf. [17], corollary 7.1d):

Farkas' lemma: Let $V$ an $m \times n$ matrix with rational entries and let $x \in \mathbb{Q}^{m}$. Then there exists $a \geq 0, a \in \mathbb{Q}^{n}$ such that $V a=x$ if and only if for all $u \in \mathbb{Q}^{m}, u^{\top} V \geq 0$ implies $u^{\top} x \geq 0$.

We can relate this to our problem in the following way. First note that the condition that for all $u \in \mathbb{Q}^{m}, u^{\top} V \geq 0$ implies $u^{\top} x \geq 0$ is equivalent to the condition that for all $u \in \mathbb{Z}^{m}, u^{\top} V \geq 0$ implies $u^{\top} x \geq 0$. Necessity is clear and sufficiency follows since if $u \in \mathbb{Q}^{m}$ and $u^{\top} V \geq 0$ then $u=c u^{\prime}$ with $c \in \mathbb{Q}, c>0$ and $u^{\prime} \in \mathbb{Z}^{m}$. Then $u^{\prime \top} V \geq 0$, so $u^{\prime \top} x \geq 0$ and multiplying by $c$ gives the required inequality.

Now consider the inequalities

$$
u^{\top} V \geq 0, \quad u \in \mathbb{Z}^{m}
$$

By writing $u_{i}=w_{i}-v_{i}, w_{i}, v_{i} \in \mathbb{N}, i=1 \ldots m$, we obtain a new system of linear Diophantine inequalities. It is not difficult to see that every solution to (3.8) can be obtained from a solution to this new system. Moreover, the new system of linear Diophantine inequalities has a finite set of fundamental solutions. These give rise to a set of fundamental solutions to (3.8) such that every solution to (3.8) is a linear combination of these fundamental solutions with non-negative integer coefficients. Call these fundamental solutions $s_{i}, i=1 \ldots k$. Thus the condition that for all $u \in \mathbb{Z}^{m}, u^{\top} V \geq 0$ implies $u^{\top} x \geq 0$ is equivalent to the condition $s_{i}^{\top} x \geq 0, i=1 \ldots k$.

Putting all this together we obtain the following variation of Farkas' lemma:

Lemma: Let $V$ be an $m \times n$ matrix with rational entries and let $x \in \mathbb{Q}^{m}$. Then there exists $a \geq 0, a \in \mathbb{Q}^{n}$ such that $V a=x$ if and only if $s_{i}^{\top} x \geq 0, i=1 \ldots k$ where $s_{i}, i=1 \ldots k$ are a fundamental set of solutions of the system $u^{\top} V \geq 0, u \in \mathbb{Z}^{m}$.

We can reformulate this Lemma over the integers in a form which is more convenient for our application: 
Proposition A: Suppose $V \in M_{m, n}(\mathbb{N})$ and let $x \in \mathbb{N}^{m}, a \in \mathbb{N}^{n}$. Then $V a=x$ if and only if $u_{i}^{\top} x=\alpha_{i}^{\top} a, i=1 \ldots k$ where $u_{i}, \alpha_{i}, i=1 \ldots k$ are a fundamental set of solutions of the system $u^{\top} V=\alpha^{\top}, u \in \mathbb{Z}^{m}, \alpha \in \mathbb{N}^{n}$.

To show this, suppose that $V a=x$ and that $u^{\top} V=\alpha^{\top}$ with $u \in \mathbb{Z}^{m}, \alpha \in \mathbb{N}^{n}$. Then $u^{\top} x=u^{\top} V a=\alpha^{\top} a$. In particular this is true for the fundamental solutions.

Conversely, suppose $u_{i}^{\top} x=\alpha_{i}^{\top} a$ for every fundamental solution. Then $u^{\top} x=\alpha^{\top} a$ for every $u \in \mathbb{Z}^{m}, \alpha \in \mathbb{N}^{n}$ such that $u^{\top} V=\alpha^{\top}$. Since $V \in M_{m, n}(\mathbb{N})$, one set of solutions to $u^{\top} V=\alpha^{\top}, u \in \mathbb{Z}^{m}, \alpha \in \mathbb{N}^{n}$ is given by taking $u$ to be a suitable unit vector and $\alpha^{\top}$ to be a row of $V$ which gives $V a=x$ as required.

To link the lemma to the situation presented above, we note that the entries $V_{i j}$ of the matrix $V$ are given here by the numbers $\epsilon_{j i}$ appearing in (3.6). Our analogue of the relation $V a=x$ describes a generic coupling and our goal is to find the defining system of inequalities underlying the existence of this coupling. The equalities $u_{i}^{\top} x=\alpha_{i}^{\top} a$ $i=1 \ldots k$ imply that $x$ satisfies $u_{i}^{\top} x \geq 0 i=1 \ldots k$ since $\alpha_{i}$ and $a$ are non-negative. In general these inequalities have solutions which are not solutions of the former equalities for any $a$. For example if $V=(2)$, then $V a=x$ is $2 a=x$ which is also the equality obtained from the second part of the Proposition A. Thus $x$ is a non-negative even integer. But the corresponding inequality is $x \geq 0$. However, we have found that for the particular systems we consider, this does not happen - as can be easily verified by computing the fundamental set of solutions to the inequalities $u_{i}^{\top} x \geq 0 i=1 \ldots k$ and verifying that they are the columns of $V$.

As a simple illustration of this construction, let us work out the example of $\widehat{s u}(2)$. We use the $\mathrm{LR}$ variables $\left\{k, \lambda_{1}, n_{11}, n_{12}\right\}$ and the corresponding grading variables $\left\{d, L_{1}, N_{11}, N_{12}\right\}$ in terms of which the elementary couplings and the corresponding vectors are

$$
\begin{array}{ll}
\widehat{E}_{0}: d & \epsilon_{0}=(1,0,0,0) \\
\widehat{E}_{1}: d L_{1} N_{12} & \epsilon_{1}=(1,1,0,1) \\
\widehat{E}_{2}: d L_{1} & \epsilon_{2}=(1,1,0,0) \\
\widehat{E}_{3}: d N_{11} & \epsilon_{3}=(1,0,1,0)
\end{array}
$$

For future reference, we display the LR tableaux of the corresponding tensor-product elementary couplings

$$
E_{1}: \square, \quad E_{2}: \square, \quad E_{3}: \quad 1
$$


To the fusion elementary couplings, we associate the vectors $\epsilon_{j}$ which form the matrix $V$ with components $V_{i j}=\epsilon_{j i}$ :

$$
V=\left(\begin{array}{llll}
1 & 1 & 1 & 1 \\
0 & 1 & 1 & 0 \\
0 & 0 & 0 & 1 \\
0 & 1 & 0 & 0
\end{array}\right)
$$

and so we have the matrix equation

$$
V a=x
$$

This equation describes a general fusion coupling. We now want to unravel the underlying system of inequalities. For this, we use Proposition A, i.e., we find the fundamental solutions of $u^{\top} V \geq 0$. This is first transformed into a set of equalities $u^{\top} V=\alpha^{\top}$ by introducing new non-negative parameters $\alpha_{i}$ :

$$
\begin{array}{ll}
u_{0}=\alpha_{0} & u_{0}+u_{1}=\alpha_{2} \\
u_{0}+u_{1}+u_{3}=\alpha_{1} & u_{0}+u_{2}=\alpha_{3}
\end{array}
$$

We next apply the vector-basis arguments (see Section 7 of [1]). Let us choose the $\alpha_{i}$ as our independent variables. (This example is somewhat misleading due to its simplicity: in general not all the $\alpha_{i}$ can be taken as the independent variables.) The dependent variables read then

$$
\begin{array}{ll}
u_{0}=\alpha_{0} & u_{2}=\alpha_{3}-\alpha_{0} \\
u_{1}=\alpha_{2}-\alpha_{0} & u_{3}=\alpha_{1}-\alpha_{2}
\end{array}
$$

The 4 basis vectors are obtained by setting successively one $\alpha_{i}$ equal to 1 and all the others equal to 0 . These vectors are written as $e_{i}$ and their entries are

$$
e_{i}=\left(u_{0}\left(\alpha_{i}=1\right), u_{1}\left(\alpha_{i}=1\right), u_{2}\left(\alpha_{i}=1\right), u_{3}\left(\alpha_{i}=1\right) ; \alpha_{0}, \alpha_{1}, \alpha_{2}, \alpha_{3}\right)
$$

With $i=0,1,2,3$, we find

$$
\begin{array}{ll}
e_{0}=(1,-1,-1,0 ; 1,0,0,0) & e_{3}=(0,0,1,0 ; 0,0,0,1) \\
e_{1}=(0,0,0,1 ; 0,1,0,0) & e_{2}=(0,1,0,-1 ; 0,0,1,0)
\end{array}
$$

These $e_{i}$ are manifestly linearly independent and they are non-negative expressions in the $\alpha_{i}$. In other words, their grading re-transcription of the above vectors (with $U_{i}$ and $\mathcal{A}_{i}$ denoting the grading variables of $u_{i}$ and $\alpha_{i}$ respectively) reads

$$
\begin{array}{ll}
\mathcal{E}_{0}=U_{0} U_{1}^{-1} U_{2}^{-1} \mathcal{A}_{0} & \mathcal{E}_{2}=U_{1} U_{3}^{-1} \mathcal{A}_{2} \\
\mathcal{E}_{1}=U_{3} \mathcal{A}_{1} & \mathcal{E}_{3}=U_{2} \mathcal{A}_{3}
\end{array}
$$


Here we see that all $\mathcal{E}_{i}$ contain positive powers of the $\mathcal{A}_{i}$ (this is not generic and it reflects the simplicity of the $s u(2)$ case). Hence, all solutions are generated freely from the nonnegative powers of the $\mathcal{E}_{i}$.

The corresponding linear system of Proposition $\mathrm{A}$ is $e_{i}(x,-a)^{\top}=0$ with $x=$ $\left(k, \lambda_{1}, n_{11}, n_{12}\right)$ and $a=\left(a_{1}, a_{2}, a_{3}, a_{4}\right)$ non-negative integers:

$$
\begin{array}{ll}
k-\lambda_{1}-n_{11}=a_{1} & \lambda_{1}-n_{12}=a_{3} \\
n_{12}=a_{2} & n_{11}=a_{4}
\end{array}
$$

which are equivalent to the inequalities:

$$
\begin{array}{ll}
k \geq \lambda_{1}+n_{11} & \lambda_{1} \geq n_{12} \\
n_{12} \geq 0 & n_{11} \geq 0
\end{array}
$$

The last three conditions define the LR basis. The first one is the additional fusion constraint.

In general, we will work the elementary solutions $e_{i}$ in their exponential version $\mathcal{E}_{i}$ to keep the notation more compact and it should be clear that the (in)equalities can be read off as easily at this level.

The construction of the $\widehat{s u}(2)$ generating function is now straightforward: since there are no relations between the elementary couplings, the generating function is simply (2.27), that is

$$
G^{\widehat{s u}(2)}=\prod_{i=0}^{3} \frac{1}{\left(1-\widehat{E}_{i}\right)}
$$

From the $k$-inequality of the $\widehat{s u}(2)$ fusion basis, we read off the threshold level of a coupling as $k_{0}=\lambda_{1}+n_{11}$, that is

$$
k_{0}=\left(\lambda_{1}+\mu_{1}+\nu_{1}\right) / 2
$$

The threshold level is also nicely coded in the LR tableaux: all elementary couplings have threshold level 1 and they all have a single column. We can then write directly that

$$
k_{0}=\# \text { columns }=\lambda_{1}+n_{11}
$$

and we recover the previous result. For an $s u(2)$ LR tableau, it is clear that the number of columns is given by this expression. More generally, for $s u(N)$, it is simple to check that the number of columns is simply

$$
\text { \#columns }=\left(\lambda+\mu+\nu, \omega_{N-1}\right)=\sum_{i=1}^{N-1} \lambda_{i}+n_{11}
$$

where $\omega_{N-1}$ is the $N-1$-th fundamental weight. 


\section{The generating function for $\widehat{s u}(3)$ fusion rules}

The $s u(3)$ tensor-product elementary couplings are:

$$
\begin{aligned}
& E_{1}=\frac{\square}{\frac{1}{2}}, \quad E_{2}=\square, \quad E_{3}=1, \quad E_{4}=\frac{\square}{1} \\
& E_{5}=\square, \quad E_{6}=\frac{1}{2}, \quad E_{7}=\frac{\square}{1}, \quad E_{8}=\frac{\square}{2} .
\end{aligned}
$$

Using the Kac-Walton formula, the threshold level of $E_{1}$ is 1 and the corresponding fusion reads

$$
\widehat{E}_{1}: \quad[0,1,0] \times[0,0,1] \supset[1,0,0]
$$

Acting on $\widehat{E}_{1}$ with $\left(a^{n}, a^{m} ; a^{n+m}\right) n, m=0,1,2$ yields the elementary couplings:

$$
\begin{array}{llll}
\widehat{E}_{0}: & {[1,0,0] \times[1,0,0] \supset[1,0,0]:} & d & (1,0,0,0,0,0,0,0) \\
\widehat{E}_{1}: & {[0,1,0] \times[0,0,1] \supset[1,0,0]:} & d L_{1} N_{12} N_{23} & (1,1,0,0,1,0,0,1) \\
\widehat{E}_{2}: & {[0,1,0] \times[1,0,0] \supset[0,1,0]:} & d L_{1} & (1,1,0,0,0,0,0,0) \\
\widehat{E}_{3}: & {[1,0,0] \times[0,1,0] \supset[0,1,0]:} & d N_{11} & (1,0,0,1,0,0,0,0) \\
\widehat{E}_{4}: & {[0,0,1] \times[0,1,0] \supset[1,0,0]:} & d L_{2} N_{13} & (1,0,1,0,0,1,0,0) \\
\widehat{E}_{5}: & {[0,0,1] \times[1,0,0] \supset[0,0,1]:} & d L_{2} & (1,0,1,0,0,0,0,0) \\
\widehat{E}_{6}: & {[1,0,0] \times[0,0,1] \supset[0,0,1]:} & d N_{11} N_{22} & (1,0,0,1,0,0,1,0) \\
\widehat{E}_{7}: & {[0,1,0] \times[0,1,0] \supset[0,0,1]:} & d L_{1} N_{12} & (1,1,0,0,1,0,0,0) \\
\widehat{E}_{8}: & {[0,0,1] \times[0,0,1] \supset[0,1,0]:} & d L_{2} N_{11} N_{23} & (1,0,1,1,0,0,0,1)
\end{array}
$$

The last column is the vector $\epsilon_{i}$ with entries $\left(k, \lambda_{1}, \lambda_{2}, n_{11}, n_{12}, n_{13}, n_{22}, n_{23}\right)$. By this procedure, we have thus recovered the affine extension of the 8 tensor-product elementary couplings and found an extra elementary coupling: $\widehat{E}_{0}$.

To derive the fusion basis, we proceed as in the $s u(2)$ case. The set of variables here is

$$
\left(x_{0}, x_{1}, \cdots, x_{7}\right)=\left(k, \lambda_{1}, \lambda_{2}, n_{11}, n_{12}, n_{13}, n_{22}, n_{23}\right)
$$


and the matrix $V$ (with columns written in the order $\widehat{E}_{0}, \cdots, \widehat{E}_{8}$ ) reads

$$
V=\left(\begin{array}{lllllllll}
1 & 1 & 1 & 1 & 1 & 1 & 1 & 1 & 1 \\
0 & 1 & 1 & 0 & 0 & 0 & 0 & 1 & 0 \\
0 & 0 & 0 & 0 & 1 & 1 & 0 & 0 & 1 \\
0 & 0 & 0 & 1 & 0 & 0 & 1 & 0 & 1 \\
0 & 1 & 0 & 0 & 0 & 0 & 0 & 1 & 0 \\
0 & 0 & 0 & 0 & 1 & 0 & 0 & 0 & 0 \\
0 & 0 & 0 & 0 & 0 & 0 & 1 & 0 & 0 \\
0 & 1 & 0 & 0 & 0 & 0 & 0 & 0 & 1
\end{array}\right)
$$

The reformulation of $u^{\top} V \geq 0$ in terms of equalities by the introduction of appropriate nonnegative parameters reads:

$$
\begin{array}{ll}
u_{0}=\alpha_{0} & u_{0}+u_{2}=\alpha_{5} \\
u_{0}+u_{1}+u_{4}+u_{7}=\alpha_{1} & u_{0}+u_{3}+u_{6}=\alpha_{6} \\
u_{0}+u_{1}=\alpha_{2} & u_{0}+u_{1}+u_{4}=\alpha_{7} \\
u_{0}+u_{3}=\alpha_{3} & u_{0}+u_{2}+u_{3}+u_{7}=\alpha_{8} \\
u_{0}+u_{2}+u_{5}=\alpha_{4} &
\end{array}
$$

We have 17 variables and 9 equations, hence 8 free variables. Let us choose them to be the $\alpha_{i}$ except for $\alpha_{5}$. Solving for the dependent variables leads to

$$
\begin{array}{ll}
u_{0}=\alpha_{0} & u_{5}=-\alpha_{0}+\alpha_{1}+\alpha_{3}+\alpha_{4}-\alpha_{7}-\alpha_{8} \\
u_{1}=-\alpha_{0}+\alpha_{2} & u_{6}=-\alpha_{3}+\alpha_{6} \\
u_{2}=-\alpha_{1}-\alpha_{3}+\alpha_{7}+\alpha_{8} & u_{7}=\alpha_{1}-\alpha_{7} \\
u_{3}=-\alpha_{0}+\alpha_{3} & \alpha_{5}=\alpha_{0}-\alpha_{1}-\alpha_{3}+\alpha_{7}+\alpha_{8} \\
u_{4}=-\alpha_{2}+\alpha_{7} &
\end{array}
$$

The basis vectors $e_{i}$ of this system are obtained by setting one of the $\alpha_{j}=1$ and all the others equal to 0 (with the understanding the $\alpha_{5}$ is excluded from this list of free variables). It appears more natural here to express them in their exponentiated version since a projection will be needed to extract the non-negative fundamental solutions. Denote by $U_{i}$ the grading variable associated to $u_{i}$ and by $\mathcal{A}_{i}$ those associated to $\alpha_{i}$, the exponential form of the basis vectors reads

$$
\begin{array}{ll}
\mathcal{E}_{0}: U_{0} U_{1}^{-1} U_{3}^{-1} U_{5}^{-1} \mathcal{A}_{0} \mathcal{A}_{5} & \mathcal{E}_{4}: U_{5} \mathcal{A}_{4} \\
\mathcal{E}_{1}: U_{2}^{-1} U_{5} U_{7} \mathcal{A}_{1} \mathcal{A}_{5}^{-1} & \mathcal{E}_{5}: U_{6} \mathcal{A}_{6} \\
\mathcal{E}_{2}: U_{1} U_{4}^{-1} \mathcal{A}_{2} & \mathcal{E}_{6}: U_{2} U_{4} U_{5}^{-1} U_{7}^{-1} \mathcal{A}_{5} \mathcal{A}_{7} \\
\mathcal{E}_{3}: U_{2}^{-1} U_{3} U_{5} U_{6}^{-1} \mathcal{A}_{3} \mathcal{A}_{5}^{-1} & \mathcal{E}_{7}: U_{2} U_{5}^{-1} \mathcal{A}_{5} \mathcal{A}_{8}
\end{array}
$$


To get the corresponding non-negative couplings, i.e., terms containing only non-negative powers of the $\mathcal{A}_{i}$, we must keep only the non-negative powers of the $\mathcal{E}_{i}$. But this is not sufficient since negative powers of $\mathcal{A}_{5}$ can appear: we need to project the free generators of the non-negative $\mathcal{E}_{i}$ powers

$$
\prod_{i=0}^{7} \frac{1}{1-\mathcal{E}_{i}}
$$

to non-negative $\mathcal{A}_{5}$ powers, using, say the MacMahon algorithm (cf. the $\Omega$ projection described in section 3 of [1]). After the projection, all the variables $\mathcal{A}_{i}$ are set equal to 1 . Here however, it is fairly easy to find out by inspection those non-negative combinations of the $\mathcal{E}_{i}$ that have non-negative $\mathcal{A}_{5}$ terms. These are

$$
\mathcal{E}_{0}, \mathcal{E}_{2}, \mathcal{E}_{4}, \mathcal{E}_{5}, \mathcal{E}_{6}, \mathcal{E}_{7}
$$

together with

$$
\begin{array}{ll}
\mathcal{E}_{0} \mathcal{E}_{1}: U_{0} U_{1}^{-1} U_{2}^{-1} U_{3}^{-1} U_{7} \mathcal{A}_{0} \mathcal{A}_{1} & \mathcal{E}_{1} \mathcal{E}_{7}: U_{7} \mathcal{A}_{1} \mathcal{A}_{8} \\
\mathcal{E}_{0} \mathcal{E}_{3}: U_{0} U_{1}^{-1} U_{2}^{-1} U_{6}^{-1} \mathcal{A}_{0} \mathcal{A}_{3} & \mathcal{E}_{3} \mathcal{E}_{6}: U_{3} U_{4} U_{6}^{-1} U_{7}^{-1} \mathcal{A}_{3} \mathcal{A}_{7} \\
\mathcal{E}_{1} \mathcal{E}_{6}: U_{4} \mathcal{A}_{1} \mathcal{A}_{7} & \mathcal{E}_{3} \mathcal{E}_{7}: U_{3} U_{6}^{-1} \mathcal{A}_{3} \mathcal{A}_{8}
\end{array}
$$

At this point, we set all $\mathcal{A}_{i}=1$. We have thus 12 elementary non-negative solutions and the corresponding inequalities are:

$$
\begin{array}{ll}
\lambda_{1} \geq n_{12} & \lambda_{2} \geq n_{13} \quad \lambda_{2}+n_{12} \geq n_{13}+n_{23} \\
n_{11} \geq n_{22} & n_{11}+n_{12} \geq n_{22}+n_{23}
\end{array}
$$

and $n_{i j} \geq 0$ (except for $n_{11} \geq 0$ which is implied by the others), which are the LR conditions for $s u(3)$. There are also three inequalities involving $k$ :

$$
\begin{aligned}
k-\lambda_{1}-\lambda_{2} & \geq n_{22} \\
k-\lambda_{1}-\lambda_{2} & \geq n_{11}-n_{23} \\
k-\lambda_{1} & \geq n_{13}+n_{11}
\end{aligned}
$$

The set of inequalities (4.12) and (4.13) represents the $\widehat{s u}(3)$ fusion basis.

Before we leave the analysis of the $\widehat{s u}(3)$ case, let us return to the set of equations (4.7). The last equality gives a relation between different $\alpha_{i}$. Actually this relation signals a relation between different sums of columns of $V$. In other words, this signals a relation 
between products of elementary couplings. Indeed, to link the last equality of (4.7) with such a relation, we recall that the labelling of the $\alpha_{i}$ is that of the elementary couplings, which are the columns of $V$. Hence, the sought for relation is simply the product form of the equality with $\alpha_{i} \mapsto \widehat{E}_{i}$ :

$$
\alpha_{1}+\alpha_{3}+\alpha_{5}=\alpha_{0}+\alpha_{7}+\alpha_{8} \mapsto \widehat{E}_{1} \widehat{E}_{3} \widehat{E}_{5}=\widehat{E}_{0} \widehat{E}_{7} \widehat{E}_{8}
$$

As there is only one relation, it is easy to find the generating function. Forbidding $\widehat{E}_{1} \widehat{E}_{3} \widehat{E}_{5}$, we get [4]

$$
\begin{array}{r}
G_{1}=\left(\prod_{\substack{i=0 \\
i \neq 1,3,5}}^{8}\left(1-\widehat{E}_{i}\right)^{-1}\right)\left(\frac{1}{\left(1-\widehat{E}_{1}\right)\left(1-\widehat{E}_{5}\right)}\right. \\
\left.+\frac{\widehat{E}_{3}}{\left(1-\widehat{E}_{3}\right)\left(1-\widehat{E}_{1}\right)}+\frac{\widehat{E}_{3} \widehat{E}_{5}}{\left(1-\widehat{E}_{5}\right)\left(1-\widehat{E}_{3}\right)}\right)
\end{array}
$$

If instead, we decide to forbid $\widehat{E}_{0} \widehat{E}_{7} \widehat{E}_{8}$, we would have

$$
\begin{array}{r}
G^{\prime}=\left(\prod_{\substack{i=0 \\
i \neq 0,7,8}}^{8}\left(1-\widehat{E}_{i}\right)^{-1}\right)\left(\frac{1}{\left(1-\widehat{E}_{0}\right)\left(1-\widehat{E}_{7}\right)}\right. \\
\left.+\frac{\widehat{E}_{8}}{\left(1-\widehat{E}_{7}\right)\left(1-\widehat{E}_{8}\right)}+\frac{\widehat{E}_{0} \widehat{E}_{8}}{\left(1-\widehat{E}_{8}\right)\left(1-\widehat{E}_{0}\right)}\right)
\end{array}
$$

and simple manipulations show that $G_{1}=G^{\prime}$. An independent proof of this generating function is presented in Appendix A.

Given the fusion basis, we can write down directly the threshold level to be

$$
k_{0}=\max \left(\lambda_{1}+\lambda_{2}+n_{11}-n_{23}, \lambda_{1}+\lambda_{2}+n_{22}, \lambda_{1}+n_{11}+n_{13}\right)
$$

This can also be extracted from the generating function as follows. A generic term of the $\widehat{s u}(3)$ generating function is $\left(\widehat{E}_{0}=d\right)$

$$
d^{\alpha} \widehat{E}_{1}^{a} \widehat{E}_{2}^{b} \widehat{E}_{3}^{c} \widehat{E}_{4}^{d} \widehat{E}_{5}^{e} \widehat{E}_{6}^{f} \widehat{E}_{7}^{g} \widehat{E}_{8}^{h}
$$

with either $a=0, c=0$ or $e=0$. In all cases the threshold level is simply

$$
k_{0}=a+b+c+d+e+f+g+h
$$


In terms of the grading variables $L_{i}$ and $N_{i j}$, the above generic term becomes

$$
d^{\alpha+k_{0}} L_{1}^{a+b+g} L_{2}^{d+e+h} N_{11}^{c+f+h} N_{12}^{a+g} N_{13}^{d} N_{22}^{f} N_{23}^{a+h}
$$

From this expression we read off the relation between the $n_{i j}$ and the variables $a, \cdots, h$. In each three cases (where one of $a, c, e$ is zero), we can then solve for the sum $k_{0}=$ $a+b+c+d+e+f+g+h$. We find

$$
\begin{array}{ll}
a=0: & k_{0}=\lambda_{1}+\lambda_{2}+n_{11}-n_{23} \\
c=0: & k_{0}=\lambda_{1}+\lambda_{2}+n_{22} \\
e=0: & k_{0}=\lambda_{1}+n_{11}+n_{13}
\end{array}
$$

This leads to the compact expression (4.17) for the $\widehat{s u}(3)$ threshold level. This is easily checked to be equivalent to the formula given in $[6,7]$ in terms of BZ triangle data (cf. section 7.1 of [1]):

$$
k_{0}=\max \left\{m_{13}+\mu_{1}+\mu_{2}, n_{13}+\nu_{1}+\nu_{2}, l_{13}+\lambda_{1}+\lambda_{2}\right\}
$$

An explicit formula for the $\widehat{s u}(3)$ fusion coefficients is written down in [19].

Notice that the threshold level is also simply encoded in the LR tableaux. Indeed, every elementary couplings has threshold level 1 and it corresponds to the number of columns except for $E_{8}$. This leads directly to the following formula for the threshold level of a general LR tableau

$$
k \geq k_{0} \equiv \# \text { columns }-\# E_{8}=\# \text { columns }-\# \begin{array}{|l|l|}
\hline & 1 \\
\hline & \\
&
\end{array}
$$

that is, $k_{0}$ is the number of columns minus the total number of $E_{8}$ that we can take out of the tableau while preserving its LR character. Consider for instance:

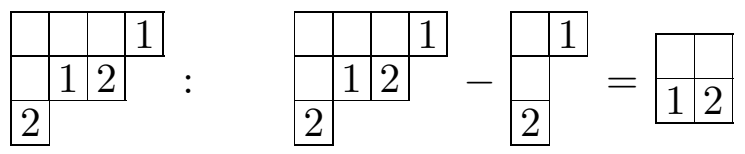

After the subtraction of one $E_{8}$, the resulting tableau is not a LR tableau: counting from right to left, we find that a 2 precedes the first 1 . Therefore, no $E_{8}$ can be removed and $k_{0}$ is given by the number of columns which is 4 . 


\section{The $\widehat{s p}(4)$ generating function}

We first recall some results obtained in [1]. The appropriate basis for the description of $s p(4)$ tensor products reads [20]:

$$
\begin{array}{ll}
\lambda_{1} \geq p & \mu_{1} \geq q \\
\lambda_{2} \geq r_{1} / 2 & \mu_{1} \geq q+r_{1}-r_{2} \\
\lambda_{2} \geq r_{1} / 2+q-p & \mu_{1} \geq p+r_{1}-r_{2} \\
\lambda_{2} \geq r_{2} / 2+q-p & \mu_{2} \geq r_{2} / 2 \\
\nu_{1}=r_{2}-r_{1}-2 p+\lambda_{1}+\mu_{1} & \nu_{2}=p-q-r_{2}+\lambda_{2}+\mu_{2}
\end{array}
$$

together with $p, q \in \mathbb{N}$ and $r_{i} \in 2 \mathbb{N}$ for $i=1,2$. A proper set of variables for a complete description of a particular tensor-product coupling is thus

$$
\left\{\lambda_{1}, \lambda_{2}, \mu_{1}, \mu_{2}, r_{1}, r_{2}, p, q\right\}
$$

(notice the absence of the $\nu_{i}$ Dynkin labels). Let the corresponding grading variables be

$$
\left\{L_{1}, L_{2}, M_{1}, M_{2}, R_{1}, R_{2}, P, Q\right\}
$$

The list of elementary coupling with their grading description is:

$$
\begin{array}{ll}
A_{1}:(0,0) \otimes(1,0) \supset(1,0) & M_{1} \\
A_{2}:(1,0) \otimes(0,0) \supset(1,0) & L_{1} \\
A_{3}:(1,0) \otimes(1,0) \supset(0,0) & L_{1} M_{1} P Q \\
B_{1}:(0,0) \otimes(0,1) \supset(0,1) & M_{2} \\
B_{2}:(0,1) \otimes(0,0) \supset(0,1) & L_{2} \\
B_{3}:(0,1) \otimes(0,1) \supset(0,0) & L_{2} M_{2} R_{1}^{2} R_{2}^{2} \\
C_{1}:(0,1) \otimes(1,0) \supset(1,0) & L_{2} M_{1} Q \\
C_{2}:(1,0) \otimes(0,1) \supset(1,0) & L_{1} M_{2} R_{2}^{2} P \\
C_{3}:(1,0) \otimes(1,0) \supset(0,1) & L_{1} M_{1} P \\
D_{1}:(2,0) \otimes(0,1) \supset(0,1) & L_{1}^{2} M_{2} R_{2}^{2} P^{2} \\
D_{2}:(0,1) \otimes(2,0) \supset(0,1) & L_{2} M_{1}^{2} R_{1}^{2} \\
D_{3}:(0,1) \otimes(0,1) \supset(2,0) & L_{2} M_{2} R_{2}^{2}
\end{array}
$$


The relation between elementary couplings are generated by

$$
\begin{array}{lll}
C_{1} C_{2}=A_{3} D_{3}, & C_{2} C_{3}=A_{1} D_{1} & C_{3} C_{1}=A_{1} A_{3} B_{2} \\
D_{1} D_{2}=B_{3} C_{3}^{2} & D_{2} D_{3}=A_{1}^{2} B_{2} B_{3} & D_{1} D_{3}=B_{2} C_{2}^{2} \\
C_{1} D_{1}=A_{3} B_{2} C_{2} & C_{2} D_{2}=A_{1} B_{3} C_{3} & C_{3} D_{3}=A_{1} B_{2} C_{2}
\end{array}
$$

To find the fusion elementary couplings, we start by computing the threshold level of $A_{1}$ by the Kac-Walton formula. It is found to be 1 . The corresponding level-1 fusion, denoted $\widehat{A}_{1}$, is thus

$$
[1,0,0] \times[0,1,0] \supset[0,1,0]
$$

We can act on it with the four pairs

$$
\left(A, A^{\prime}\right)=\{(1,1),(a, a),(a, 1),(1, a)\}
$$

We obtain in this way two copies of $\widehat{A}_{1}$ and two copies of $\widehat{C}_{1}$, the level-1 extension of $C_{1}$. Similarly, $A_{2}$, and $A_{3}$ are found to have level 1 and this implies the same result for $C_{2}, C_{3}$. $B_{1}$ is also found to have threshold level 1 . Acting on it with the above sequence of outer automorphisms leads successively to $B_{1}, B_{2}, B_{3}$ and a new coupling, $\widehat{E}_{0}$ :

$$
\widehat{E}_{0}: \quad[1,0,0] \times[1,0,0] \supset[1,0,0]
$$

Finally, $D_{1}, D_{2}$ and $D_{3}$ have threshold level 2 and they are all fixed with respect to the action of the outer-automorphism group. The set $\left\{\widehat{A}_{i}, \widehat{B}_{i}, \widehat{C}_{i}, \widehat{D}_{i}, \widehat{E}_{0}\right\}$ is thus our candidate complete set of fusion elementary couplings, whose explicit expression in terms of grading variables is read from their tensor-product relative with the addition of an appropriate factors of $d$.

Having obtained the fusion elementary couplings, we now work out the corresponding fusion basis. Introduce the set of variables

$$
\left(x_{0}, x_{1}, \cdots, x_{8}\right)=\left(k, \lambda_{1}, \lambda_{2}, \mu_{1}, \mu_{2}, r_{1}, r_{2}, p, q\right)
$$

This fixes the ordering of the rows of $V$. The matrix $V$ is built from the columns which form the different elementary couplings in the order $\widehat{E}_{0}, \widehat{A}_{1}, \cdots, \widehat{D}_{3}$ :

$$
V=\left(\begin{array}{lllllllllllll}
1 & 1 & 1 & 1 & 1 & 1 & 1 & 1 & 1 & 1 & 2 & 2 & 2 \\
0 & 0 & 1 & 1 & 0 & 0 & 0 & 0 & 1 & 1 & 2 & 0 & 0 \\
0 & 0 & 0 & 0 & 0 & 1 & 1 & 1 & 0 & 0 & 0 & 1 & 1 \\
0 & 1 & 0 & 1 & 0 & 0 & 0 & 1 & 0 & 1 & 0 & 2 & 0 \\
0 & 0 & 0 & 0 & 1 & 0 & 1 & 0 & 1 & 0 & 1 & 0 & 1 \\
0 & 0 & 0 & 0 & 0 & 0 & 2 & 0 & 0 & 0 & 0 & 2 & 0 \\
0 & 0 & 0 & 0 & 0 & 0 & 2 & 0 & 2 & 0 & 2 & 0 & 2 \\
0 & 0 & 0 & 1 & 0 & 0 & 0 & 0 & 1 & 1 & 2 & 0 & 0 \\
0 & 0 & 0 & 1 & 0 & 0 & 0 & 1 & 0 & 0 & 0 & 0 & 0
\end{array}\right)
$$


The transcription of the inequalities $u^{\top} V \geq 0$ into the equalities $u^{\top} V=\alpha^{\top}$ takes the following form

$$
\begin{array}{ll}
u_{0}=\alpha_{0} & u_{0}+u_{2}+u_{3}+u_{8}=\alpha_{7} \\
u_{0}+u_{3}=\alpha_{1} & u_{0}+u_{1}+u_{4}+2 u_{6}+u_{7}=\alpha_{8} \\
u_{0}+u_{1}=\alpha_{2} & u_{0}+u_{1}+u_{3}+u_{7}=\alpha_{9} \\
u_{0}+u_{1}+u_{3}+u_{7}+u_{8}=\alpha_{3} & 2 u_{0}+2 u_{1}+u_{4}+2 u_{6}+2 u_{7}=\alpha_{10} \\
u_{0}+u_{4}=\alpha_{4} & 2 u_{0}+u_{2}+2 u_{3}+2 u_{5}=\alpha_{11} \\
u_{0}+u_{2}=\alpha_{5} & 2 u_{0}+u_{2}+u_{4}+2 u_{6}=\alpha_{12} \\
u_{0}+u_{2}+u_{4}+2 u_{5}+2 u_{6}=\alpha_{6} &
\end{array}
$$

Solving for the dependent variables $u_{i}, \alpha_{j}, i=0, \cdots, 8$ and $j=6,7,8,9$ gives

$$
\begin{array}{ll}
u_{0}=\alpha_{0} & u_{7}=\frac{1}{2}\left(\alpha_{0}-2 \alpha_{2}+\alpha_{5}+\alpha_{10}-\alpha_{12}\right) \\
u_{1}=-\alpha_{0}+\alpha_{2} & u_{8}=\frac{1}{2}\left(\alpha_{0}-2 \alpha_{1}+2 \alpha_{3}-\alpha_{5}-\alpha_{10}+\alpha_{12}\right) \\
u_{2}=-\alpha_{0}+\alpha_{5} & \alpha_{6}=-2 \alpha_{1}-\alpha_{5}+\alpha_{11}+\alpha_{12} \\
u_{3}=-\alpha_{0}+\alpha_{1} & \alpha_{7}=\frac{1}{2}\left(-\alpha_{0}+2 \alpha_{3}+\alpha_{5}-\alpha_{10}+\alpha_{12}\right) \\
u_{4}=-\alpha_{0}+\alpha_{4} & \alpha_{8}=\frac{1}{2}\left(-\alpha_{0}-\alpha_{5}+\alpha_{10}+\alpha_{12}\right) \\
u_{5}=\frac{1}{2}\left(\alpha_{0}-2 \alpha_{1}-\alpha_{5}+\alpha_{11}\right) & \alpha_{9}=\frac{1}{2}\left(-\alpha_{0}+2 \alpha_{1}+\alpha_{5}+\alpha_{10}-\alpha_{12}\right) \\
u_{6}=\frac{1}{2}\left(-\alpha_{4}-\alpha_{5}+\alpha_{12}\right) &
\end{array}
$$

As usual, the basis vectors $e_{i}$ of this system are obtained by setting one of the $\alpha_{i}=1$ and all the others equal to 0 , excluding $\alpha_{6}, \cdots, \alpha_{9}$. We will give their exponentiated version, where as before, we denote by $U_{i}$ the grading variable associated to $u_{i}$ and by $\mathcal{A}_{i}$ those associated to $\alpha_{i}$ :

$$
\begin{aligned}
& \mathcal{E}_{0}: U_{0} U_{1}^{-1} U_{2}^{-1} U_{3}^{-1} U_{4}^{-1} U_{5}^{1 / 2} U_{7}^{1 / 2} U_{8}^{1 / 2} \mathcal{A}_{0} \mathcal{A}_{7}^{-1 / 2} \mathcal{A}_{8}^{-1 / 2} \mathcal{A}_{9}^{-1 / 2} \\
& \mathcal{E}_{1}: U_{3} U_{5}^{-1} U_{8}^{-1} \mathcal{A}_{1} \mathcal{A}_{6}^{-2} \mathcal{A}_{9} \\
& \mathcal{E}_{2}: U_{1} U_{7}^{-1} \mathcal{A}_{2} \\
& \mathcal{E}_{3}: U_{8} \mathcal{A}_{3} \mathcal{A}_{7} \\
& \mathcal{E}_{4}: U_{4} U_{6}^{-1 / 2} \mathcal{A}_{4} \\
& \mathcal{E}_{5}: U_{2} U_{5}^{-1 / 2} U_{6}^{-1 / 2} U_{7}^{1 / 2} U_{8}^{-1 / 2} \mathcal{A}_{5} \mathcal{A}_{6}^{-1} \mathcal{A}_{7}^{1 / 2} \mathcal{A}_{8}^{-1 / 2} \mathcal{A}_{9}^{1 / 2} \\
& \mathcal{E}_{6}: U_{7}^{1 / 2} U_{8}^{-1 / 2} \mathcal{A}_{7}^{-1 / 2} \mathcal{A}_{8}^{1 / 2} \mathcal{A}_{9}^{1 / 2} \mathcal{A}_{10} \\
& \mathcal{E}_{7}: U_{5}^{1 / 2} \mathcal{A}_{6} \mathcal{A}_{11} \\
& \mathcal{E}_{8}: U_{6}^{1 / 2} U_{7}^{-1 / 2} U_{8}^{1 / 2} \mathcal{A}_{6} \mathcal{A}_{7}^{1 / 2} \mathcal{A}_{8}^{1 / 2} \mathcal{A}_{9}^{-1 / 2} \mathcal{A}_{12}
\end{aligned}
$$


Next we keep only those combinations of the $\mathcal{E}_{i}$ that contain only non-negative integer powers of the $\mathcal{A}_{i}$. This projection is not so simple to work out by inspection. We thus need to use a more systematic procedure:

Consider a general expansion of the form $\prod_{i}\left(1-\mathcal{E}_{i}\right)^{-1}$ and in a generic term of the form $\prod_{i} \mathcal{E}_{i}^{\epsilon_{i}^{\prime}}$, let us collect the number of $\mathcal{A}_{i}$ factors (denote by $a_{i}$ their exponents). Of course we are only interested in those $\mathcal{A}_{i}$ that appear with negative powers, namely $i=6,7,8,9$. Their powers can be read off from the $\mathcal{A}_{i}$ in (5.13) and this yields the following expressions:

$$
\begin{aligned}
& a_{6}=-2 \epsilon_{1}^{\prime}-\epsilon_{5}^{\prime}+\epsilon_{7}^{\prime}+\epsilon_{8}^{\prime} \geq 0 \\
& 2 a_{7}=-\epsilon_{0}^{\prime}+2 \epsilon_{3}^{\prime}+\epsilon_{5}^{\prime}-\epsilon_{6}^{\prime}+\epsilon_{8}^{\prime} \geq 0 \\
& 2 a_{8}=-\epsilon_{0}^{\prime}-\epsilon_{5}^{\prime}+\epsilon_{6}^{\prime}+\epsilon_{8}^{\prime} \geq 0 \\
& 2 a_{9}=-\epsilon_{0}^{\prime}+2 \epsilon_{1}^{\prime}+\epsilon_{5}^{\prime}+\epsilon_{6}^{\prime}-\epsilon_{8}^{\prime} \geq 0
\end{aligned}
$$

(These equations should be compared with the last four of (5.12), with $\alpha_{i} \rightarrow \epsilon_{i}^{\prime}, i \leq 5$ and $\alpha_{i} \rightarrow e_{i-4}^{\prime}$ for $i \geq 10$ ). We then look for the elementary solutions of this system of inequalities. There are 4 elementary solutions with $\epsilon_{0}^{\prime} \neq 0$. Their grading reformulation reads

$$
\mathcal{E}_{0} \mathcal{E}_{1} \mathcal{E}_{6} \mathcal{E}_{8}^{2} \quad \mathcal{E}_{0} \mathcal{E}_{1} \mathcal{E}_{7} \mathcal{E}_{8} \quad \mathcal{E}_{0} \mathcal{E}_{5} \mathcal{E}_{6} \mathcal{E}_{8} \quad \mathcal{E}_{0} \mathcal{E}_{3} \mathcal{E}_{6}
$$

Denote their vector-reformulation respectively as $e_{i}$ with $i=0,1,2,3$, then the conditions $e_{i} x \geq 0$ yield, in the above order

$$
\begin{aligned}
& k \geq \lambda_{1}+\lambda_{2}+\mu_{2}+r_{1} / 2-r_{2} \\
& k \geq \lambda_{1}+\lambda_{2}+\mu_{2}-r_{2} / 2 \\
& k \geq \lambda_{1}+\mu_{1}+\mu_{2}-p \\
& k \geq \lambda_{1}+\lambda_{2}+\mu_{1}+\mu_{2}-p-q-r_{1} / 2
\end{aligned}
$$

The other elementary solutions are

$$
\begin{aligned}
& \mathcal{E}_{2}, \quad \mathcal{E}_{3}, \quad \mathcal{E}_{4}, \quad \mathcal{E}_{7}, \quad \mathcal{E}_{5} \mathcal{E}_{8}, \quad \mathcal{E}_{6} \mathcal{E}_{8}, \quad \mathcal{E}_{1} \mathcal{E}_{8}^{2}, \quad \mathcal{E}_{1} \mathcal{E}_{7}^{2}, \\
& \mathcal{E}_{3} \mathcal{E}_{6}^{2}, \quad \mathcal{E}_{5} \mathcal{E}_{6} \mathcal{E}_{7}, \quad \mathcal{E}_{5} \mathcal{E}_{6}^{2} \mathcal{E}_{8}, \quad \mathcal{E}_{1} \mathcal{E}_{6}^{2} \mathcal{E}_{8},
\end{aligned}
$$

and the resulting inequalities reproduce the whole set of BZ inequalities (5.1) with the positivity requirement on $r_{i}, p$ and $q$ (together with $\mu_{1} \geq q+\frac{1}{2}\left(r_{1}-r_{2}\right)$ which is implied by the other ones). 
Let us return to the last four equations in (5.12). As mentioned in connection to the $\widehat{s u}(3)$ case, they indicate the 'basic relations': the correspondence between the $\alpha_{i}$ and the elementary couplings being fixed by the ordering of the columns of $V$ (e.g., $\alpha_{3} \mapsto \widehat{A}_{3}$ and $\left.\alpha_{7} \mapsto \widehat{C}_{1}\right)$. The relations correspond then respectively to

$$
\begin{array}{ll}
\widehat{A}_{1}^{2} \widehat{B}_{2} \widehat{B}_{3}=\widehat{D}_{2} \widehat{D}_{3} & \widehat{E}_{0} \widehat{C}_{1}^{2} \widehat{D}_{1}=\widehat{A}_{3}^{2} \widehat{B}_{2} \widehat{D}_{3} \\
\widehat{E}_{0} \widehat{B}_{2} \widehat{C}_{2}^{2}=\widehat{D}_{1} \widehat{D}_{3} & \widehat{E}_{0} \widehat{C}_{3}^{2} \widehat{D}_{3}=\widehat{A}_{1}^{2} \widehat{B}_{2} \widehat{D}_{1}
\end{array}
$$

The first and third relations appear in the list (5.5). All other linear relations in the set (5.5) can be obtained from products of the above four, allowing for the cancellations of common factors. For instance, consider the product of the left factors of the second and third relations; equating this with the product of the right factors yields

$$
\widehat{E}_{0}^{2} \widehat{B}_{2} \widehat{C}_{1}^{2} \widehat{C}_{2}^{2} \widehat{D}_{1}=\widehat{A}_{3}^{2} \widehat{B}_{2} \widehat{D}_{1} \widehat{D}_{3}^{2}
$$

Cancelling the $\widehat{B}_{2} \widehat{D}_{1}$ terms and taking the square root gives

$$
\widehat{E}_{0} \widehat{C}_{1} \widehat{C}_{2}=\widehat{A}_{3} \widehat{D}_{3}
$$

which is the affine extension of the relation $C_{1} C_{2}=A_{3} D_{3}$. All other linear relations can be obtained in a similar way.

We can write the $\widehat{s p}(4)$ generating function in the compact form

$$
\begin{aligned}
G & =\bar{E}_{0} \bar{B}_{1} \bar{B}_{2} \bar{B}_{3}\left[\bar{A}_{1} \bar{A}_{2} \bar{A}_{3} \bar{C}_{1} \bar{C}_{2} \bar{C}_{3}\left(1-\widehat{A}_{1} \widehat{A}_{3} \widehat{B}_{2}\right)+\widehat{D}_{1} \bar{D}_{1} \bar{A}_{2} \bar{A}_{3} \bar{C}_{2} \bar{C}_{3}\right. \\
& \left.+\widehat{D}_{3} \bar{D}_{3} \bar{A}_{1} \bar{A}_{2} \bar{C}_{1} \bar{C}_{2}+\widehat{D}_{2} \bar{D}_{2} \bar{A}_{1} \bar{A}_{2} \bar{A}_{3} \bar{C}_{1} \bar{C}_{3}\left(1-\widehat{A}_{1} \widehat{A}_{3} \widehat{B}_{2}\right)\right]
\end{aligned}
$$

where $\bar{Q}$ is defined as

$$
\bar{Q}=\frac{1}{1-\widehat{Q}}
$$

This can be re-expressed under a manifestly positive form as follows

$$
\begin{aligned}
G & =\bar{E}_{0} \bar{A}_{1} \bar{A}_{2} \bar{B}_{1} \bar{B}_{2} \bar{B}_{3} \bar{C}_{1} \bar{C}_{2} \bar{C}_{3}+\bar{E}_{0} \widehat{A}_{3} \bar{A}_{2} \bar{A}_{3} \bar{B}_{1} \bar{B}_{2} \bar{B}_{3} \bar{C}_{1} \bar{C}_{2} \bar{C}_{3} \\
& +\bar{E}_{0} \widehat{A}_{1} \widehat{A}_{3} \bar{A}_{1} \bar{A}_{2} \bar{A}_{3} \bar{B}_{1} \bar{B}_{3} \bar{C}_{1} \bar{C}_{2} \bar{C}_{3}+\bar{E}_{0} \widehat{D}_{1} \bar{A}_{2} \bar{A}_{3} \bar{B}_{1} \bar{B}_{2} \bar{B}_{3} \bar{C}_{2} \bar{C}_{3} \bar{D}_{1} \\
& +\bar{E}_{0} \widehat{D}_{3} \bar{A}_{1} \bar{A}_{2} \bar{B}_{1} \bar{B}_{2} \bar{B}_{3} \bar{C}_{1} \bar{C}_{2} \bar{D}_{3}+\bar{E}_{0} \widehat{D}_{2} \bar{A}_{1} \bar{A}_{2} \bar{B}_{1} \bar{B}_{2} \bar{B}_{3} \bar{C}_{1} \bar{C}_{3} \bar{D}_{2} \\
& +\bar{E}_{0} \widehat{A}_{3} \widehat{D}_{2} \bar{A}_{2} \bar{A}_{3} \bar{B}_{1} \bar{B}_{2} \bar{B}_{3} \bar{C}_{1} \bar{C}_{3} \bar{D}_{2}+\bar{E}_{0} \widehat{A}_{1} \widehat{A}_{3} \widehat{D}_{2} \bar{A}_{1} \bar{A}_{2} \bar{A}_{3} \bar{B}_{1} \bar{B}_{3} \bar{C}_{1} \bar{C}_{3} \bar{D}_{2}
\end{aligned}
$$


We should stress that this is essentially a new result. A generating function for $\widehat{s p}(4)$ fusion rules was given in [21]; the approach, however, was ad hoc and the result was not related to any known basis.

As before the information concerning the threshold level that can be deduced from the fusion basis inequalities (5.16) can also be obtained directly from the generating function. A generic term of the $\widehat{s p}(4)$ generating function (5.23) reads

$$
d^{\alpha} \widehat{A}_{1}^{a} \widehat{A}_{2}^{b} \widehat{A}_{3}^{c} \widehat{B}_{1}^{d} \widehat{B}_{2}^{e} \widehat{B}_{3}^{f} \widehat{C}_{1}^{g} \widehat{C}_{2}^{h} \widehat{C}_{3}^{i} \widehat{D}_{1}^{j} \widehat{D}_{2}^{k} \widehat{D}_{3}^{l}
$$

Its threshold level is (since all these factors have a single power of $d$ except for the three $\left.\widehat{D}_{i}=d^{2} D_{i}\right)$ :

$$
k_{0}=a+b+c+d+e+f+g+h+i+2 j+2 k+2 l
$$

Now express the elementary couplings in terms of dummy variables $\left\{L_{1}, L_{2}, M_{1}, M_{2}, R_{1}, R_{2}, P, Q\right\}$ whose exponent are the BZ basis data, respectively $\left\{\lambda_{1}, \lambda_{2}, \mu_{1}, \mu_{2}, r_{1}, r_{2}, p, q\right\}$ :

$$
\begin{array}{lll}
A_{1}=M_{1} & A_{2}=L_{1} & A_{3}=L_{1} M_{1} P Q \\
B_{1}=M_{2} & B_{2}=L_{2} & B_{3}=L_{2} M_{2} R_{1}^{2} R_{2}^{2} \\
C_{1}=L_{2} M_{1} Q & C_{2}=L_{1} M_{2} R_{2}^{2} P & C_{3}=L_{1} M_{1} P \\
D_{1}=L_{1}^{2} M_{2} R_{2}^{2} P^{2} & D_{2}=L_{2} M_{1}^{2} R_{1}^{2} & D_{3}=L_{2} M_{2} R_{2}^{2}
\end{array}
$$

Next, consider each term of the generating function (5.23) and solve for $k_{0}$ in terms of the basis variables. Surprisingly there are only four different formulas for $k_{0}$. The expressions corresponding to the different terms of (5.23) are:

$$
\begin{array}{ll}
\text { terms 1,5,6: } & k_{0}=\lambda_{1}+\lambda_{2}+\mu_{1}+\mu_{2}-p-q-r_{1} / 2 \\
\text { terms 3,8: } & k_{0}=\lambda_{1}+\mu_{1}+\mu_{2}-p \\
\text { term } 7: & k_{0}=\lambda_{1}+\lambda_{2}+\mu_{2}+r_{1} / 2-r_{2} \\
\text { terms 2, 4: } & k_{0}=\lambda_{1}+\lambda_{2}+\mu_{2}-r_{2} / 2
\end{array}
$$

Therefore, the threshold formula is the maximum value of these four values or equivalently

$$
k_{0}=\lambda_{1}+\lambda_{2}+\mu_{1}+\mu_{2}-\min \left(p+q+r_{1} / 2, \lambda_{2}+p, \mu_{1}-r_{1} / 2+r_{2}, \mu_{1}+r_{2} / 2\right)
$$

Notice that by rewriting $k \geq k_{0}$, we recover from $(5.27)$ the 4 inequalities $(5.16)$. 
The system of inequalities (5.1) can be transformed into a system of equations by setting $r_{1} / 2=s_{1}$ and $r_{2} / 2=s_{2}$ and introducing the integers $a_{i}$ (cf. section 7.5 of [1]):

$$
\begin{array}{ll}
\lambda_{1}=p+a_{1} & \nu_{2}=a_{4}+a_{8} \\
\lambda_{2}=s_{1}+a_{2} & a_{2}+p=a_{3}+q \\
\mu_{1}=q+a_{5} & a_{3}+s_{1}=a_{4}+s_{2} \\
\mu_{2}=s_{2}+a_{8} & a_{5}+2 s_{2}=a_{6}+2 s_{1} \\
\nu_{1}=a_{1}+a_{7} & a_{6}+q=a_{7}+p
\end{array}
$$

As shown in [1], this leads to the following diamond-type graphical representation of the tensor product:

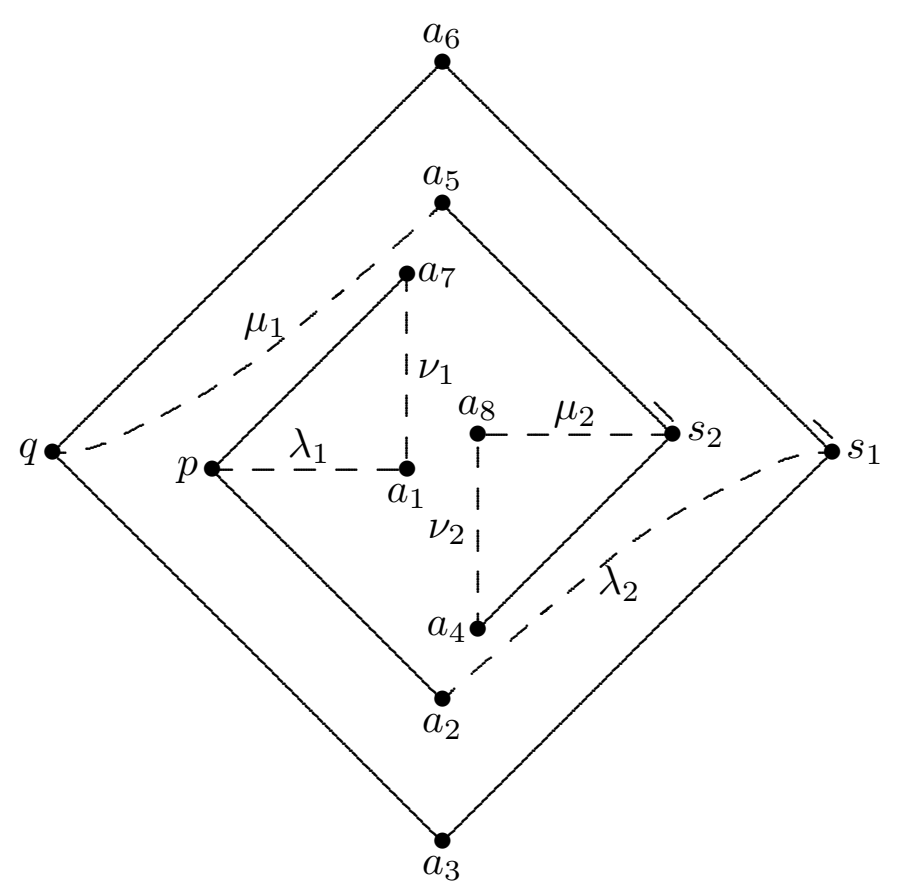

Dotted lines relate those two points that compose the label indicated beside it and opposite continuous lines are constrained to be equal, with the length of a line being defined as the sum of its extremal points except for the lines delimited by the points $\left(a_{6}, s_{1}\right)$ and $\left(a_{5}, s_{2}\right)$ where the point $s_{i}$ is counted twice (the little bar besides $s_{1}$ and $s_{2}$ being a reminder of this particularity). For those lines, the constraint reads $a_{6}+2 s_{1}=a_{5}+2 s_{2}$. Given a triple $s p(4)$ product, the number of such diamonds that can be drawn with only non-negative entries gives its multiplicity. 
In terms of these data, the expression for the threshold level (5.27) look somewhat more symmetrical: the four expressions in (5.27) correspond respectively to the following terms:

$$
k_{0}=a_{1}+a_{8}+\max \left\{a_{4}+a_{7}+s_{1}, a_{5}+q+s_{2}, a_{4}+q+s_{1}, a_{4}+q+s_{2}\right\}
$$

\section{The $\widehat{s u}(4)$ generating function}

Written directly in terms of LR tableaux, the $s u(4)$ elementary solutions are:

$$
\begin{aligned}
& A_{1}=\frac{\sqrt{1}}{\frac{1}{3}}, \quad A_{2}=\frac{\square}{\frac{1}{1}}, \quad A_{3}=\square, \quad B_{1}=\frac{1}{2}, \quad B_{2}=\frac{\square}{\frac{1}{2}}, \quad B_{3}=\square, \\
& C_{1}=1, \quad C_{2}=\frac{\square}{\frac{1}{3}}, \quad C_{3}=\square, \quad D_{1}^{\prime}=\frac{\square}{\square}, \quad D_{2}^{\prime}=\frac{\square}{1}, \quad D_{3}^{\prime}=\frac{\square}{\frac{1}{2}},
\end{aligned}
$$

and

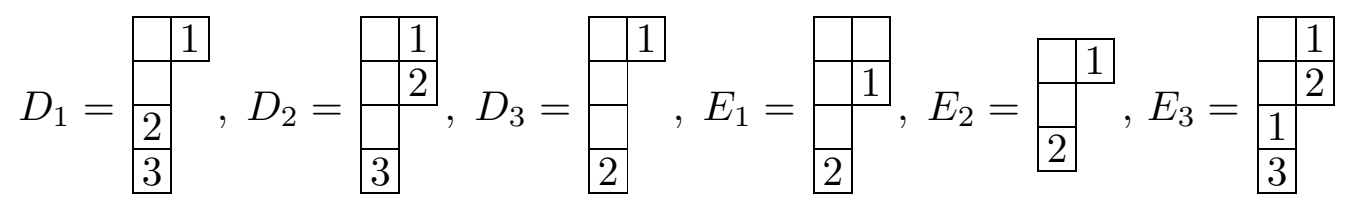

The relations are $[22,23]$ :

$$
\begin{array}{ll}
D_{j}^{\prime} D_{k}=C_{i} E_{i} & D_{j} D_{k}^{\prime}=B_{i} C_{j} C_{k} \\
D_{i} E_{i}=C_{j} B_{k} D_{k} & D_{i}^{\prime} E_{i}=B_{j} D_{j}^{\prime} C_{k}=B_{k} D_{k} D_{k}^{\prime}
\end{array}
$$

with $i, j, k$ a cyclic permutation of $1,2,3$.

Consider now the construction of the set of fusion elementary couplings using outerautomorphism completeness. Start with $A_{1}:(0,0,0) \otimes(0,0,1) \supset(0,0,1)$, this has threshold level 1. Acting on it with

$$
\left(A, A^{\prime}\right)=\left(a^{n}, a^{m}\right), \quad n, m=0,1,2,3
$$

where

$$
a\left[\lambda_{0}, \lambda_{1} \lambda_{2}, \lambda_{3}\right]=\left[\lambda_{3}, \lambda_{0} \lambda_{1}, \lambda_{2}\right]
$$


we generate the affine extension of the whole set $A_{i}, B_{i}, C_{i}, D_{i}, D_{i}^{\prime}$, which thus all have threshold level 1, together with the scalar coupling $\widehat{E}_{0}=[1,0,0,0] \times[1,0,0,0] \supset[1,0,0,0]$. Finally, the affine extension of $E_{1}$ arises first at level 2: $[0,1,0,1] \times[1,0,1,0] \supset[1,0,1,0]$. The three weights in this coupling are fixed under the action of $A=a^{2}$. Hence, we need only to consider

$$
\left(A, A^{\prime}\right)=\{(1,1),(a, a),(a, 1),(1, a)\}
$$

and this leads respectively to $\widehat{E}_{1}, \widehat{E}_{2}, \widehat{E}_{3}$, which all have $k_{0}=2$ and a new elementary coupling $\widehat{F}=[0,1,0,1] \times[0,1,0,1] \supset[0,1,0,1]$ (first discovered in [7]). Notice that at the level of tensor-products, $\widehat{F}$ is a composite product $C_{1} C_{2} C_{3}$. But if it were still composite for fusions, it would necessarily have level 3 since $k_{0}\left(C_{1} C_{2} C_{3}\right)=3$. This is the reason why $\widehat{F}$ must be regarded as a new elementary coupling.

The whole set of fusion elementary couplings is:

$$
\begin{array}{rlrl}
\widehat{A}_{1} & =[1,0,0,0] \times[0,0,0,1] \supset[0,0,0,1] & & \widehat{D}_{1}^{\prime}=[0,0,1,0] \times[0,1,0,0] \supset[0,0,0,1] \\
\widehat{A}_{2}=[0,0,0,1] \times[0,1,0,0] \supset[1,0,0,0] & & \widehat{D}_{2}^{\prime}=[0,1,0,0] \times[0,1,0,0] \supset[0,0,1,0] \\
\widehat{A}_{3}=[0,1,0,0] \times[1,0,0,0] \supset[0,1,0,0] & & \widehat{D}_{3}^{\prime}=[0,1,0,0] \times[0,0,1,0] \supset[0,0,0,1] \\
\widehat{B}_{1}=[0,0,0,0] \times[0,0,1,0] \supset[0,0,1,0] & & \widehat{D}_{1}=[0,0,1,0] \times[0,0,0,1] \supset[0,1,0,0] \\
\widehat{B}_{2}=[0,0,1,0] \times[0,0,1,0] \supset[1,0,0,0] & & \widehat{D}_{2}=[0,0,0,1] \times[0,0,0,1] \supset[0,0,1,0] \\
\widehat{B}_{3}=[0,0,1,0] \times[1,0,0,0] \supset[0,0,1,0] & & \widehat{D}_{3}=[0,0,0,1] \times[0,0,1,0] \supset[0,1,0,0] \\
\widehat{C}_{1}=[1,0,0,0] \times[0,1,0,0] \supset[0,1,0,0] & & \widehat{E}_{1}=[0,1,0,1] \times[1,0,1,0] \supset[1,0,1,0] \\
\widehat{C}_{2}=[0,1,0,0] \times[0,0,0,1] \supset[1,0,0,0] & & \widehat{E}_{2}=[1,0,1,0] \times[1,0,1,0] \supset[0,1,0,1] \\
\widehat{C}_{3}=[0,0,0,1] \times[1,0,0,0] \supset[0,0,0,1] & & \widehat{E}_{3}=[1,0,1,0] \times[0,1,0,1] \supset[1,0,1,0]
\end{array}
$$

together with two couplings that have no elementary finite relative:

$$
\widehat{E}_{0}=[1,0,0,0] \times[1,0,0,0] \supset[1,0,0,0] \quad \widehat{F}=[0,1,0,1] \times[0,1,0,1] \supset[0,1,0,1]
$$

The tensor-product relations are modified by the appropriate insertions of $d$ or $\widehat{E}_{0}$ factors in order to put them at the same threshold level:

$$
\begin{array}{lll}
\widehat{E}_{0} \widehat{D}_{j}^{\prime} \widehat{D}_{k}=\widehat{C}_{i} \widehat{E}_{i} & \widehat{E}_{0} \widehat{D}_{j} \widehat{D}_{k}^{\prime}=\widehat{B}_{i} \widehat{C}_{j} \widehat{C}_{k} & \widehat{E}_{i} \widehat{E}_{j}=\widehat{E}_{0} \widehat{B}_{k} \widehat{D}_{k} \widehat{D}_{k}^{\prime} \\
\widehat{D}_{i} \widehat{E}_{i}=\widehat{C}_{j} \widehat{B}_{k} \widehat{D}_{k} & \widehat{D}_{i}^{\prime} \widehat{E}_{i}=\widehat{B}_{j} \widehat{D}_{j}^{\prime} \widehat{C}_{k} & \widehat{E}_{0} \widehat{F}=\widehat{C}_{1} \widehat{C}_{2} \widehat{C}_{3}
\end{array}
$$

with $i, j, k$ a cyclic permutation of $1,2,3$. 
To get the $\widehat{s u}(4)$ basis, we first write down the $V$ matrix, whose columns are the vectorial transcription of the fusion elementary couplings written in terms of the grading variables. The column ordering corresponds to $\widehat{E}_{0}, \widehat{A}_{i}, \widehat{B}_{i}, \widehat{C}_{i}, \widehat{D}_{i}^{\prime}, \widehat{D}_{i}, \widehat{E}_{i}, \widehat{F}$ with $i=1,2,3$. The rows are labelled by the $\mathrm{LR}$ variables $\left(k, \lambda_{1}, \lambda_{2}, \lambda_{3}, n_{11}, n_{12}, n_{13}, n_{14}, n_{22}, n_{23}, n_{24}, n_{33}, n_{34}\right)$. The matrix $V$ is thus

$$
V=\left(\begin{array}{llllllllllllllllllll}
1 & 1 & 1 & 1 & 1 & 1 & 1 & 1 & 1 & 1 & 1 & 1 & 1 & 1 & 1 & 1 & 2 & 2 & 2 & 2 \\
0 & 0 & 0 & 1 & 0 & 0 & 0 & 0 & 1 & 0 & 0 & 1 & 1 & 0 & 0 & 0 & 1 & 0 & 0 & 1 \\
0 & 0 & 0 & 0 & 0 & 1 & 1 & 0 & 0 & 0 & 1 & 0 & 0 & 1 & 0 & 0 & 0 & 1 & 1 & 0 \\
0 & 0 & 1 & 0 & 0 & 0 & 0 & 0 & 0 & 1 & 0 & 0 & 0 & 0 & 1 & 1 & 1 & 0 & 0 & 1 \\
0 & 1 & 0 & 0 & 1 & 0 & 0 & 1 & 0 & 0 & 0 & 0 & 0 & 1 & 1 & 1 & 0 & 1 & 1 & 1 \\
0 & 0 & 0 & 0 & 0 & 0 & 0 & 0 & 1 & 0 & 0 & 1 & 1 & 0 & 0 & 0 & 1 & 0 & 0 & 1 \\
0 & 0 & 0 & 0 & 0 & 1 & 0 & 0 & 0 & 0 & 1 & 0 & 0 & 0 & 0 & 0 & 0 & 0 & 1 & 0 \\
0 & 0 & 1 & 0 & 0 & 0 & 0 & 0 & 0 & 0 & 0 & 0 & 0 & 0 & 0 & 0 & 0 & 0 & 0 & 0 \\
0 & 1 & 0 & 0 & 1 & 0 & 0 & 0 & 0 & 0 & 0 & 0 & 0 & 0 & 1 & 0 & 0 & 0 & 1 & 0 \\
0 & 0 & 0 & 0 & 0 & 0 & 0 & 0 & 1 & 0 & 0 & 0 & 1 & 1 & 0 & 0 & 0 & 1 & 0 & 1 \\
0 & 0 & 0 & 0 & 0 & 1 & 0 & 0 & 0 & 0 & 0 & 0 & 0 & 0 & 0 & 1 & 1 & 0 & 0 & 0 \\
0 & 1 & 0 & 0 & 0 & 0 & 0 & 0 & 0 & 0 & 0 & 0 & 0 & 0 & 0 & 0 & 0 & 0 & 0 & 0 \\
0 & 0 & 0 & 0 & 0 & 0 & 0 & 0 & 1 & 0 & 0 & 0 & 0 & 1 & 1 & 0 & 0 & 0 & 1 & 1
\end{array}\right)
$$

With $u=\left(u_{0}, \cdots, u_{12}\right)$, the equations $u^{\top} V \geq 0$ can be transformed into equalities by introducing the variables $\alpha_{i}$ :

$$
\begin{array}{ll}
u_{0}=\alpha_{0} & u_{0}+u_{2}+u_{6}=\alpha_{10} \\
u_{0}+u_{4}+u_{8}+u_{11}=\alpha_{1} & u_{0}+u_{1}+u_{5}=\alpha_{11} \\
u_{0}+u_{3}+u_{7}=\alpha_{2} & u_{0}+u_{1}+u_{5}+u_{9}=\alpha_{12} \\
u_{0}+u_{1}=\alpha_{3} & u_{0}+u_{2}+u_{4}+u_{9}+u_{12}=\alpha_{13} \\
u_{0}+u_{4}+u_{8}=\alpha_{4} & u_{0}+u_{3}+u_{4}+u_{8}+u_{12}=\alpha_{14} \\
u_{0}+u_{2}+u_{6}+u_{10}=\alpha_{5} & u_{0}+u_{3}+u_{4}+u_{10}=\alpha_{15} \\
u_{0}+u_{2}=\alpha_{6} & 2 u_{0}+u_{1}+u_{3}+u_{5}+u_{10}=\alpha_{16} \\
u_{0}+u_{4}=\alpha_{7} & 2 u_{0}+u_{2}+u_{4}+u_{9}=\alpha_{17} \\
u_{0}+u_{1}+u_{5}+u_{9}+u_{12}=\alpha_{8} & 2 u_{0}+u_{2}+u_{4}+u_{6}+u_{8}+u_{12}=\alpha_{18} \\
u_{0}+u_{3}=\alpha_{9} & 2 u_{0}+u_{1}+u_{3}+u_{4}+u_{5}+u_{9}+u_{12}=\alpha_{19}
\end{array}
$$

We have 13 free variables; let us choose them to be the $\alpha_{i}$ for $i=0, \cdots, 12$. Solving 
for the dependent variables leads to

$$
\begin{array}{lll}
u_{0}=\alpha_{0} & u_{5}=-\alpha_{3}+\alpha_{11} & u_{10}=\alpha_{5}-\alpha_{10} \\
u_{1}=-\alpha_{0}+\alpha_{3} & u_{6}=-\alpha_{6}+\alpha_{10} & u_{11}=\alpha_{1}-\alpha_{4} \\
u_{2}=-\alpha_{0}+\alpha_{6} & u_{7}=\alpha_{2}-\alpha_{9} & u_{12}=\alpha_{8}-\alpha_{12} \\
u_{3}=-\alpha_{0}+\alpha_{9} & u_{8}=\alpha_{4}-\alpha_{7} & \\
u_{4}=-\alpha_{0}+\alpha_{7} & u_{9}=-\alpha_{11}+\alpha_{12} &
\end{array}
$$

together with

$$
\begin{array}{ll}
\alpha_{13}=-\alpha_{0}+\alpha_{6}+\alpha_{7}+\alpha_{8}-\alpha_{11} & \alpha_{17}=\alpha_{6}+\alpha_{7}-\alpha_{11}+\alpha_{12} \\
\alpha_{14}=-\alpha_{0}+\alpha_{4}+\alpha_{8}+\alpha_{9}-\alpha_{12} & \alpha_{18}=\alpha_{4}+\alpha_{8}+\alpha_{10}-\alpha_{12} \\
\alpha_{15}=-\alpha_{0}+\alpha_{5}+\alpha_{7}+\alpha_{9}-\alpha_{10} & \alpha_{19}=-\alpha_{0}+\alpha_{7}+\alpha_{8}+\alpha_{9} \\
\alpha_{16}=\alpha_{5}+\alpha_{9}-\alpha_{10}+\alpha_{11} &
\end{array}
$$

Now, by setting successively $\alpha_{i}=1$ for $i=0, \cdots, 12$ and the others equal to 0 , we generate the following set of basis vectors:

$$
\begin{array}{ll}
\mathcal{E}_{0}=d N_{11}^{-1} L_{1}^{-1} L_{2}^{-1} L_{3}^{-1} \mathcal{A}_{0} \mathcal{A}_{13}^{-1} \mathcal{A}_{14}^{-1} \mathcal{A}_{15}^{-1} \mathcal{A}_{19}^{-1} & \mathcal{E}_{6}=N_{13}^{-1} L_{2} \mathcal{A}_{6} \mathcal{A}_{13} \mathcal{A}_{17} \\
\mathcal{E}_{1}=N_{33} \mathcal{A}_{1} & \mathcal{E}_{7}=N_{11} N_{22}^{-1} L_{2} \mathcal{A}_{7} \mathcal{A}_{13} \mathcal{A}_{15} \mathcal{A}_{17} \mathcal{A}_{19} \\
\mathcal{E}_{2}=N_{14} \mathcal{A}_{2} & \mathcal{E}_{8}=N_{34} \mathcal{A}_{8} \mathcal{A}_{13} \mathcal{A}_{14} \mathcal{A}_{18} \mathcal{A}_{19} \\
\mathcal{E}_{3}=N_{12}^{-1} L_{1} \mathcal{A}_{3} & \mathcal{E}_{9}=N_{14}^{-1} L_{3} \mathcal{A}_{9} \mathcal{A}_{14} \mathcal{A}_{15} \mathcal{A}_{16} \mathcal{A}_{19} \\
\mathcal{E}_{4}=N_{22}^{-1} N_{33} \mathcal{A}_{4} \mathcal{A}_{14} \mathcal{A}_{18} & \mathcal{E}_{10}=N_{13} N_{24}^{-1} \mathcal{A}_{10} \mathcal{A}_{15}^{-1} \mathcal{A}_{16}^{-1} \mathcal{A}_{18} \\
\mathcal{E}_{5}=N_{24} \mathcal{A}_{5} \mathcal{A}_{15} \mathcal{A}_{16} & \mathcal{E}_{11}=N_{12} N_{23}^{-1} \mathcal{A}_{11} \mathcal{A}_{13}^{-1} \mathcal{A}_{16} \mathcal{A}_{17}^{-1} \\
& \mathcal{E}_{12}=N_{23} N_{34}^{-1} \mathcal{A}_{12} \mathcal{A}_{14}^{-1} \mathcal{A}_{17} \mathcal{A}_{18}^{-1}
\end{array}
$$

We must now look for those combinations that contain only non-negative powers of the $\mathcal{A}_{i}$. Since each $\mathcal{E}_{i}$ contains at least one positive power of $\mathcal{A}_{i}$, these must be obtained from positive combinations of the $\mathcal{E}_{j}$. To find them, it is convenient to proceed as in the analysis of $\widehat{s p}(4)$. Denote by $a_{i}$ the number of $\mathcal{A}_{i}$ factors in a general term $\prod \mathcal{E}_{i}^{\epsilon_{i}^{\prime}}$ of the free expansion of the $\mathcal{E}_{i}$ in non-negative powers we get (equivalently, we can read off the $\mathcal{A}_{i}$ from (6.14)):

$$
\begin{array}{ll}
a_{13}=-\epsilon_{0}^{\prime}+\epsilon_{6}^{\prime}+\epsilon_{7}^{\prime}+\epsilon_{8}^{\prime}-\epsilon_{11}^{\prime} & a_{17}=\epsilon_{6}^{\prime}+\epsilon_{7}^{\prime}-\epsilon_{11}^{\prime}+\epsilon_{12}^{\prime} \\
a_{14}=-\epsilon_{0}^{\prime}+\epsilon_{4}^{\prime}+\epsilon_{8}^{\prime}+\epsilon_{9}^{\prime}-\epsilon_{12}^{\prime} & a_{18}=\epsilon_{4}^{\prime}+\epsilon_{8}^{\prime}+\epsilon_{10}^{\prime}-\epsilon_{12}^{\prime} \\
a_{15}=-\epsilon_{0}^{\prime}+\epsilon_{5}^{\prime}+\epsilon_{7}^{\prime}+\epsilon_{9}^{\prime}-\epsilon_{10}^{\prime} & a_{19}=-\epsilon_{0}^{\prime}+\epsilon_{7}^{\prime}+\epsilon_{8}^{\prime}+\epsilon_{9}^{\prime} \\
a_{16}=\epsilon_{5}^{\prime}+\epsilon_{9}^{\prime}-\epsilon_{10}^{\prime}+\epsilon_{11}^{\prime} &
\end{array}
$$


These relations are to be compared with (6.13). We thus look for elementary solutions of the system $a_{i} \geq 0$ for $\epsilon_{i}^{\prime}$ non-negative integers.

The full list of composites that involve $\mathcal{E}_{0}$ - these are those that generate $k$-dependent constraints - is

$$
\begin{aligned}
& \mathcal{E}_{0} \mathcal{E}_{4} \mathcal{E}_{7}, \quad \mathcal{E}_{0} \mathcal{E}_{8} \mathcal{E}_{9}, \quad \mathcal{E}_{0} \mathcal{E}_{6} \mathcal{E}_{9}, \quad \mathcal{E}_{0} \mathcal{E}_{9} \mathcal{E}_{8}, \quad \mathcal{E}_{0} \mathcal{E}_{7} \mathcal{E}_{9}, \quad \mathcal{E}_{0} \mathcal{E}_{5} \mathcal{E}_{8}, \\
& \mathcal{E}_{0} \mathcal{E}_{7} \mathcal{E}_{8} \mathcal{E}_{11}, \quad \mathcal{E}_{0} \mathcal{E}_{7} \mathcal{E}_{9} \mathcal{E}_{12}, \quad \mathcal{E}_{0} \mathcal{E}_{7} \mathcal{E}_{9} \mathcal{E}_{10}, \quad \mathcal{E}_{0} \mathcal{E}_{5} \mathcal{E}_{8} \mathcal{E}_{12} \mathcal{E}_{4} \quad \mathcal{E}_{0}^{2} \mathcal{E}_{7} \mathcal{E}_{8} \mathcal{E}_{9}
\end{aligned}
$$

The constraints are $\left(\mathcal{E}_{i}\right.$ specifies the vector $e_{j}$ such that the inequality is $\left.e_{j} x \geq 0\right)$ :

$$
\begin{array}{ll}
\mathcal{E}_{0} \mathcal{E}_{4} \mathcal{E}_{7}: & k \geq \lambda_{1}+\lambda_{2}+\lambda_{3}+n_{33} \\
\mathcal{E}_{0} \mathcal{E}_{8} \mathcal{E}_{9}: & k \geq \lambda_{1}+\lambda_{2}+n_{11}+n_{14}-n_{34} \\
\mathcal{E}_{0} \mathcal{E}_{6} \mathcal{E}_{9}: & k \geq \lambda_{1}+n_{11}+n_{13}+n_{14} \\
\mathcal{E}_{0} \mathcal{E}_{7} \mathcal{E}_{8}: & k \geq \lambda_{1}+\lambda_{2}+\lambda_{3}+n_{22}-n_{34} \\
\mathcal{E}_{0} \mathcal{E}_{7} \mathcal{E}_{9}: & k \geq \lambda_{1}+\lambda_{2}+n_{14}+n_{22} \\
\mathcal{E}_{0} \mathcal{E}_{5} \mathcal{E}_{8}: & k \geq \lambda_{1}+\lambda_{2}+\lambda_{3}+n_{11}-n_{24}-n_{34} \\
\mathcal{E}_{0} \mathcal{E}_{7} \mathcal{E}_{8} \mathcal{E}_{11}: & k \geq \lambda_{1}+\lambda_{2}+\lambda_{3}-n_{12}+n_{22}+n_{23}-n_{34} \\
\mathcal{E}_{0} \mathcal{E}_{7} \mathcal{E}_{9} \mathcal{E}_{10}: & k \geq \lambda_{1}+\lambda_{2}+n_{14}-n_{13}+n_{22}+n_{24} \\
\mathcal{E}_{0} \mathcal{E}_{8} \mathcal{E}_{9} \mathcal{E}_{12}: & k \geq \lambda_{1}+\lambda_{2}+n_{11}+n_{14}-n_{23} \\
\mathcal{E}_{0}^{2} \mathcal{E}_{7} \mathcal{E}_{8} \mathcal{E}_{9}: & 2 k \geq 2 \lambda_{1}+2 \lambda_{2}+\lambda_{3}+n_{14}+n_{22}+n_{11}-n_{34}
\end{array}
$$

When these inequalities are re-expressed in terms of BZ triangle data, they reproduce the threshold formula presented in [7].

The $\mathcal{E}_{0}$-independent elementary solutions, namely

$$
\begin{aligned}
& \mathcal{E}_{1}, \quad \mathcal{E}_{2}, \quad \mathcal{E}_{3}, \quad \mathcal{E}_{4}, \quad \mathcal{E}_{5}, \quad \mathcal{E}_{6}, \quad \mathcal{E}_{7}, \quad \mathcal{E}_{8}, \quad \mathcal{E}_{9} \\
& \mathcal{E}_{6} \mathcal{E}_{11}, \quad \mathcal{E}_{4} \mathcal{E}_{12}, \quad \mathcal{E}_{7} \mathcal{E}_{11}, \quad \mathcal{E}_{8} \mathcal{E}_{12}, \quad \mathcal{E}_{9} \mathcal{E}_{10}, \quad \mathcal{E}_{5} \mathcal{E}_{10}, \\
& \mathcal{E}_{8} \mathcal{E}_{11} \mathcal{E}_{12}, \quad \mathcal{E}_{9} \mathcal{E}_{10} \mathcal{E}_{12}, \quad \mathcal{E}_{7} \mathcal{E}_{10} \mathcal{E}_{11}
\end{aligned}
$$

yield the standard LR inequalities:

$$
\begin{array}{ll}
\lambda_{1} \geq n_{12} & n_{11} \geq n_{22} \\
\lambda_{2} \geq n_{13} & n_{11}+n_{12} \geq n_{22}+n_{23} \\
\lambda_{2}+n_{12} \geq n_{13}+n_{23} & n_{11}+n_{12}+n_{13} \geq n_{22}+n_{23}+n_{24} \\
\lambda_{3} \geq n_{14} & n_{22} \geq n_{33} \\
\lambda_{3}+n_{13} \geq n_{14}+n_{24} & n_{22}+n_{23} \geq n_{33}+n_{34} \\
\lambda_{3}+n_{13}+n_{23} \geq n_{14}+n_{24}+n_{34} &
\end{array}
$$


and $n_{i j} \geq 0$, except for $n_{11} \geq 0$ and $n_{22} \geq 0$ which are implied by the above equations.

As in the $\widehat{s p}(4)$ case, we can check that the relations (6.13) code the 'basic linear relations' of the model. Indeed, the 7 relations read from (6.13) are

$$
\begin{array}{ll}
\widehat{E}_{0} \widehat{D}_{1} \widehat{D}_{2}^{\prime}=\widehat{B}_{3} \widehat{C}_{1} \widehat{C}_{2} & \widehat{E}_{1} \widehat{D}_{1}^{\prime}=\widehat{B}_{2} \widehat{C}_{3} \widehat{D}_{2}^{\prime} \\
\widehat{E}_{0} \widehat{D}_{2} \widehat{D}_{3}^{\prime}=\widehat{B}_{1} \widehat{C}_{2} \widehat{C}_{3} & \widehat{E}_{2} \widehat{D}_{2}^{\prime}=\widehat{B}_{3} \widehat{C}_{1} \widehat{D}_{3}^{\prime} \\
\widehat{E}_{0} \widehat{D}_{3} \widehat{D}_{1}^{\prime}=\widehat{B}_{2} \widehat{C}_{1} \widehat{C}_{3} & \widehat{E}_{3} \widehat{D}_{3}^{\prime}=\widehat{B}_{1} \widehat{C}_{3} \widehat{D}_{1}^{\prime} \\
\widehat{E}_{0} \widehat{F}=\widehat{C}_{1} \widehat{C}_{2} \widehat{C}_{3} &
\end{array}
$$

and these are the generators of all the $\widehat{s u}(4)$ linear relations.

In order to construct the $\widehat{s u}(4)$ generating function, we must choose a term ordering. We fix the ordering as follows:

$$
\begin{aligned}
& \left\{L_{1}, L_{2}, L_{3}, N_{11}, N_{12}, N_{13}, N_{14}, N_{22}, N_{23}, N_{24}, N_{33}, N_{34}, d,\right. \\
& \left.\widehat{E}_{1}, \widehat{E}_{2}, \widehat{E}_{3}, \widehat{B}_{1}, \widehat{B}_{2}, \widehat{B}_{3}, \widehat{C}_{1}, \widehat{C}_{2}, \widehat{C}_{3}, \widehat{A}_{1}, \widehat{A}_{2}, \widehat{A}_{3}, \widehat{D}_{1}, \widehat{D}_{2}, \widehat{D}_{3}, \widehat{D}_{1}^{\prime}, \widehat{D}_{2}^{\prime}, \widehat{D}_{3}^{\prime}, \widehat{E}_{0}, \widehat{F}\right\}
\end{aligned}
$$

Grobner basis methods yield the forbidden products:

$$
\begin{aligned}
& \left\{\widehat{E}_{i} \widehat{E}_{j}, \widehat{D}_{i}^{\prime} \widehat{E}_{i}, \widehat{D}_{i} \widehat{E}_{i}, \widehat{C}_{i} \widehat{E}_{i}, \widehat{B}_{i} \widehat{C}_{j} \widehat{C}_{k}, \widehat{B}_{2} \widehat{C}_{3} \widehat{D}_{1} \widehat{D}_{2}^{\prime}, \widehat{B}_{1} \widehat{C}_{3} \widehat{D}_{1} \widehat{D}_{2}^{\prime}, \widehat{B}_{1} \widehat{C}_{2} \widehat{D}_{3} \widehat{D}_{1}^{\prime}\right. \\
& \left.\widehat{E}_{0} \widehat{B}_{1} \widehat{D}_{1} \widehat{D}_{3} \widehat{D}_{1}^{\prime} \widehat{D}_{2}^{\prime}, \widehat{F} \widehat{E}_{i}, \widehat{F} \widehat{B}_{i}, \widehat{C}_{1} \widehat{C}_{2} \widehat{C}_{3}, \widehat{F} \widehat{C}_{1} \widehat{C}_{2} \widehat{C}_{3}\right\}
\end{aligned}
$$

The different terms of the generating function are fully specified by their denominator (the numerators are introduced to avoid over-counting):

$$
\begin{aligned}
& \widehat{E}_{0} \widehat{A}_{1} \widehat{A}_{2} \widehat{A}_{3} \widehat{D}_{1} \widehat{D}_{2} \widehat{D}_{3} \widehat{D}_{1}^{\prime} \widehat{D}_{2}^{\prime} \widehat{D}_{3}^{\prime} \widehat{C}_{1} \widehat{C}_{2} \widehat{F}, \quad \widehat{E}_{0} \widehat{A}_{1} \widehat{A}_{2} \widehat{A}_{3} \widehat{B}_{2} \widehat{B}_{3} \widehat{C}_{2} \widehat{C}_{3} \widehat{D}_{2} \widehat{D}_{3} \widehat{D}_{2}^{\prime} \widehat{D}_{3}^{\prime} \widehat{E}_{1} \widehat{F} \\
& \widehat{E}_{0} \widehat{A}_{1} \widehat{A}_{2} \widehat{A}_{3} \widehat{B}_{1} \widehat{B}_{2} \widehat{B}_{3} \widehat{C}_{2} \widehat{D}_{1} \widehat{D}_{2} \widehat{D}_{1}^{\prime} \widehat{D}_{2}^{\prime} \widehat{E}_{3} \widehat{F}, \quad \widehat{E}_{0} \widehat{A}_{1} \widehat{A}_{2} \widehat{A}_{3} \widehat{B}_{1} \widehat{B}_{2} \widehat{B}_{3} \widehat{C}_{1} \widehat{D}_{1} \widehat{D}_{2} \widehat{D}_{1}^{\prime} \widehat{D}_{2}^{\prime} \widehat{E}_{3} \widehat{F}, \\
& \widehat{E}_{0} \widehat{A}_{1} \widehat{A}_{2} \widehat{A}_{3} \widehat{B}_{2} \widehat{C}_{1} \widehat{C}_{2} \widehat{D}_{1} \widehat{D}_{2} \widehat{D}_{3} \widehat{D}_{1}^{\prime} \widehat{D}_{2}^{\prime} \widehat{D}_{3}^{\prime}, \quad \widehat{E}_{0} \widehat{A}_{1} \widehat{A}_{2} \widehat{A}_{3} \widehat{B}_{1} \widehat{B}_{2} \widehat{C}_{1} \widehat{C}_{2} \widehat{D}_{1} \widehat{D}_{2} \widehat{D}_{1}^{\prime} \widehat{D}_{2}^{\prime} \widehat{D}_{3}^{\prime} \text {, } \\
& \widehat{E}_{0} \widehat{A}_{1} \widehat{A}_{2} \widehat{A}_{3} \widehat{B}_{1} \widehat{B}_{2} \widehat{C}_{1} \widehat{C}_{2} \widehat{D}_{1} \widehat{D}_{2} \widehat{D}_{3} \widehat{D}_{2}^{\prime} \widehat{D}_{3}^{\prime}, \quad \widehat{E}_{0} \widehat{A}_{1} \widehat{A}_{2} \widehat{A}_{3} \widehat{B}_{3} \widehat{C}_{1} \widehat{C}_{3} \widehat{D}_{1} \widehat{D}_{2} \widehat{D}_{3} \widehat{D}_{1}^{\prime} \widehat{D}_{2}^{\prime} \widehat{D}_{3}^{\prime} \text {, } \\
& \widehat{E}_{0} \widehat{A}_{1} \widehat{A}_{2} \widehat{A}_{3} \widehat{B}_{1} \widehat{B}_{3} \widehat{C}_{1} \widehat{C}_{3} \widehat{D}_{2} \widehat{D}_{3} \widehat{D}_{1}^{\prime} \widehat{D}_{2}^{\prime} \widehat{D}_{3}^{\prime}, \quad \widehat{E}_{0} \widehat{A}_{1} \widehat{A}_{2} \widehat{A}_{3} \widehat{B}_{1} \widehat{B}_{3} \widehat{C}_{1} \widehat{C}_{3} \widehat{D}_{1} \widehat{D}_{2} \widehat{D}_{1}^{\prime} \widehat{D}_{2}^{\prime} \widehat{D}_{3}^{\prime} \text {, } \\
& \widehat{E}_{0} \widehat{A}_{1} \widehat{A}_{2} \widehat{A}_{3} \widehat{B}_{1} \widehat{B}_{3} \widehat{C}_{1} \widehat{C}_{3} \widehat{D}_{1} \widehat{D}_{2} \widehat{D}_{3} \widehat{D}_{2}^{\prime} \widehat{D}_{3}^{\prime}, \quad \widehat{E}_{0} \widehat{A}_{1} \widehat{A}_{2} \widehat{A}_{3} \widehat{B}_{1} \widehat{B}_{3} \widehat{C}_{1} \widehat{C}_{3} \widehat{D}_{1} \widehat{D}_{2} \widehat{D}_{3} \widehat{D}_{1}^{\prime} \widehat{D}_{3}^{\prime} \text {, } \\
& \widehat{A}_{1} \widehat{A}_{2} \widehat{A}_{3} \widehat{B}_{1} \widehat{B}_{3} \widehat{C}_{1} \widehat{C}_{3} \widehat{D}_{1} \widehat{D}_{2} \widehat{D}_{3} \widehat{D}_{1}^{\prime} \widehat{D}_{2}^{\prime} \widehat{D}_{3}^{\prime}, \quad \widehat{A}_{1} \widehat{A}_{2} \widehat{A}_{3} \widehat{B}_{1} \widehat{B}_{2} \widehat{B}_{3} \widehat{C}_{1} \widehat{D}_{1} \widehat{D}_{2} \widehat{D}_{3} \widehat{D}_{1}^{\prime} \widehat{D}_{2}^{\prime} \widehat{D}_{3}^{\prime} \text {, } \\
& \widehat{E}_{0} \widehat{A}_{1} \widehat{A}_{2} \widehat{A}_{3} \widehat{B}_{2} \widehat{B}_{3} \widehat{C}_{1} \widehat{D}_{1} \widehat{D}_{2} \widehat{D}_{3} \widehat{D}_{1}^{\prime} \widehat{D}_{2}^{\prime} \widehat{D}_{3}^{\prime}, \quad \widehat{E}_{0} \widehat{A}_{1} \widehat{A}_{2} \widehat{A}_{3} \widehat{B}_{1} \widehat{B}_{2} \widehat{B}_{3} \widehat{C}_{1} \widehat{D}_{2} \widehat{D}_{3} \widehat{D}_{1}^{\prime} \widehat{D}_{2}^{\prime} \widehat{D}_{3}^{\prime} \text {, } \\
& \widehat{E}_{0} \widehat{A}_{1} \widehat{A}_{2} \widehat{A}_{3} \widehat{B}_{1} \widehat{B}_{2} \widehat{B}_{3} \widehat{C}_{1} \widehat{D}_{1} \widehat{D}_{2} \widehat{D}_{1}^{\prime} \widehat{D}_{2}^{\prime} \widehat{D}_{3}^{\prime}, \quad \widehat{E}_{0} \widehat{A}_{1} \widehat{A}_{2} \widehat{A}_{3} \widehat{B}_{1} \widehat{B}_{2} \widehat{B}_{3} \widehat{C}_{1} \widehat{D}_{1} \widehat{D}_{2} \widehat{D}_{3} \widehat{D}_{2}^{\prime} \widehat{D}_{3}^{\prime} \text {, } \\
& \widehat{E}_{0} \widehat{A}_{1} \widehat{A}_{2} \widehat{A}_{3} \widehat{B}_{1} \widehat{B}_{2} \widehat{B}_{3} \widehat{C}_{1} \widehat{D}_{1} \widehat{D}_{2} \widehat{D}_{3} \widehat{D}_{1}^{\prime} \widehat{D}_{3}^{\prime} \text {, }
\end{aligned}
$$


Finally, note that the expression of threshold levels in terms of tableau data, that is, the analogue of the $\widehat{s u}(2,3)$ formulas written previously is clearly

$$
k_{0}=\# \text { columns }-\max \left\{\# D_{1}+\# D_{2}+\# D_{3}+\# C_{1} C_{2} C_{3}\right\}
$$

since the $D_{i}^{\prime} s$ have level 1 but two columns and $C_{1} C_{2} C_{3}$ has level 2 and three columns (corresponding to the $\widehat{F}$ fusion coupling.)

\section{Conclusion and open problems}

We have obtained the fusion generating function for $\widehat{s u}(3,4)$ and $\widehat{s p}(4)$ using the conjectural existence of a fusion basis. In the $\widehat{s u}(3)$ case a first-principle derivation (presented in Appendix A) provides an independent proof of the results, thus a partial confirmation of the conjectures and the correctness of the underlying fusion basis. Moreover, different tests of the $\widehat{s p}(4)$ and $\widehat{s u}(4)$ generating functions, presented in Appendix A, also support our conjectures and the fusion basis constructions. En passant, we point out that the search for the complete $\widehat{s u}(N)$ level-rank symmetric function introduced in Appendix A is a quest that deserves further studies.

Although the theme of this paper is the construction of fusion generating functions, our most important result is the unravelling of the fusion basis concept, for which we have provided concrete examples. The main open problem is to find a fundamental and Lie algebraic way of deriving the fusion basis (analogous to the Berenstein-Zelevinsky conjectures [20]). We observe that the number of $k$-type inequalities increases rather quickly with the rank of the algebra: 1 for $\widehat{s u}(2), 3$ for $\widehat{s u}(3), 4$ for $\widehat{s p}(4)$ and 10 for $\widehat{s u}(4)$. More specifically we would like to find arguments to justify the homogeneity property (on the other hand, the linearity appears to be a generic property, a direct consequence of the Kac-Walton algorithm).

With regard to the automorphism completeness conjecture we note that for simplicity (and because the discussion is to a large extent devoted to $\widehat{s u}(N)$ for which the outerautomorphism group is rather large) we have focused on the outer-automorphism group as the essential symmetry. It is natural to extend the conjecture to the full symmetry group of fusion coefficients. However, we should stress is that the outer-automorphism conjecture is just a convenient tool. If the conjecture (or its natural extension to the full 
fusion symmetry group) turns out to be wrong, there are other avenues that could yield the complete set of fusion elementary couplings.

In the present work, the only information on fusion data that has been extracted, out of the fusion basis or the fusion generating function, is the expression for the threshold level in terms of the basis variables. But there are certainly more data that can be lifted. For example, given a triple product with multiplicity $m$, to which there correspond $m$ values of the threshold levels, we could ask for the expression, in terms of the Dynkin labels, of the minimum and maximum values of $k_{0}$. It is easy to write down some explicit expressions for particular fusion coefficients.

The reformulation of the problem of computing fusion rules in terms of a fusion basis solves, in principle, the quest for a combinatorial method since it reduces a fusion computation to solving inequalities. But we expect that we have not found an optimal solution to the quest for an efficient combinatorial description.

\section{Acknowledgement}

L.B. would like to thank P. Dargis for his crucial, albeit involuntary, rôle in bringing Farkas' lemma to his attention.

\section{Appendix A. Independent verifications of the fusion generating functions}

The $\widehat{s u}(3)$ fusion generating function is not presented here for the first time; it ap-

peared originally in [4]. A sketch of its proof was presented in [24] without details. In this section we present a complete proof of the $\widehat{s u}(3)$ generating function for fusion rules; in addition, we describe some independent checks confirming the validity of the $\widehat{s u}(4)$ and $\widehat{s p}(4)$ fusion generating functions given in sections 6.3 and 7.3. The first check that we present uses Giambelli-type formulas. These can be viewed as equalities of corresponding expressions in the character rings. Since the $\widehat{s u}(n)$ and $\widehat{s p}(n)$ fusion rings are quotients of the classical character rings (see [25] and references therein), these formulas continue to hold for fusion products. For $\widehat{s u}(4)$, we present another non-trivial check based on a level-rank duality argument. 
A.1. Determinantal formula and the 'composition' method: deriving the su(3) generating function for tensor products

The Giambelli formula, or more generally, determinantal formulae which give expressions for group characters as determinants, provide another method for calculating fusion generating functions in terms of simpler generating functions. This uses the technique of 'composition' of generating functions described previously in section 2.3 of [1].

The $s u(3)$ Giambelli formula expresses a general representation in terms of a difference of products of representations with a single non-zero Dynkin label, i.e.,

$$
\left(\lambda_{1}, \lambda_{2}\right)=\left(\lambda_{1}+\lambda_{2}, 0\right) \otimes\left(\lambda_{2}, 0\right)-\left(\lambda_{1}+\lambda_{2}+1,0\right) \otimes\left(\lambda_{2}-1,0\right)
$$

This can be rewritten in determinantal form as follows

$$
\left(\lambda_{1}, \lambda_{2}\right)=\operatorname{det}\left(\begin{array}{cc}
\left(\lambda_{1}+\lambda_{2}, 0\right) & \left(\lambda_{2}-1,0\right) \\
\left(\lambda_{1}+\lambda_{2}+1,0\right) & \left(\lambda_{2}, 0\right)
\end{array}\right)
$$

Consider first the generating function $G_{1}\left(L_{1}, L_{2}, M_{1}, R_{1}, R_{2}\right)$ which is the generating function for products of the form: $\left(\lambda_{1}, \lambda_{2}\right) \otimes\left(\mu_{1}, 0\right)$. Its explicit form is

$$
G_{1}=\frac{1}{\left(1-L_{1} N_{1}\right)\left(1-L_{2} N_{2}\right)\left(1-L_{2} M_{1}\right)\left(1-M_{1} N_{1}\right)\left(1-L_{1} M_{1} N_{2}\right)}
$$

It is obtained by setting $M_{2}=0$ in the complete tensor-product generating function (cf. section 2.5 in [1]). Our point here is not to re-derive $G_{1}$ from first principles but simply to show how we can reconstruct the complete generating function out of the partial information contained in $G_{1}$. In the fusion case, we will indicate how the analogue of $G_{1}$ can be obtained, preventing the argument from being circular.

From two copies of $G_{1}$ we form the composite generating function $G_{2}$ :

$$
G_{2}\left(L_{1}, L_{2}, M_{1}, M_{2}, N_{1}, N_{2}\right)=\stackrel{R}{=} G_{1}\left(L_{1}, L_{2}, M_{1}, R_{1}, R_{2}\right) G_{1}\left(R_{1}^{-1}, R_{2}^{-1}, M_{2}, N_{1}, N_{2}\right)
$$

which is the generating function for products of the form

$$
\left(\lambda_{1}, \lambda_{2}\right) \otimes\left(\mu_{1}, 0\right) \otimes\left(\mu_{2}, 0\right)
$$

Note that the generating function for products

$$
\left(\lambda_{1}, \lambda_{2}\right) \otimes\left(\mu_{1}+1,0\right) \otimes\left(\mu_{2}-1,0\right)
$$


is $M_{2} M_{1}^{-1} G_{2}$ and so, by (A.1), the generating function for products $\left(\lambda_{1}, \lambda_{2}\right) \otimes\left(\mu_{1}, \mu_{2}\right)$ is:

$$
G_{3}=\stackrel{M}{\Omega}_{\geq}^{\Omega}\left(G_{2}-M_{2} M_{1}^{-1} G_{2}\right)
$$

The coefficient of $M_{1}^{\mu_{1}} M_{2}^{\mu_{2}}$ is the multiplicity of the representation with Dynkin labels $\left(\mu_{1}-\mu_{2}, \mu_{2}\right)$ in the product

$$
\left(\lambda_{1}, \lambda_{2}\right) \otimes\left[\left(\mu_{1}, 0\right) \otimes\left(\mu_{2}, 0\right)-\left(\mu_{1}+1,0\right) \otimes\left(\mu_{2}-1,0\right)\right]
$$

To change to variables which carry the Dynkin labels we make the substitution $M_{2} \mapsto$ $M_{2} M_{1}^{-1}$, so that $M_{1}$ now carries the first Dynkin label. This introduces negative powers of $M_{1}$, corresponding to products (A.8) with $\mu_{1}<\mu_{2}$, which are not required. So we must keep only non-negative degree terms in $M_{1}$ to obtain the final generating function. Denote the resulting expression as $G_{4}\left(L_{1}, L_{2}, M_{1}, M_{2}, N_{1}, N_{2}\right)$; it reads

$$
\begin{aligned}
G_{4}= & \frac{\left(1-L_{1} L_{2} M_{1} M_{2} N_{1} N_{2}\right)}{\left(1-L_{1} N_{1}\right)\left(1-L_{1} M_{2}\right)\left(1-L_{2} M_{1}\right)\left(1-L_{2} N_{2}\right)} \\
& \times \frac{1}{\left(1-M_{2} N_{2}\right)\left(1-M_{1} N_{1}\right)\left(1-L_{1} M_{1} N_{2}\right)\left(1-L_{2} M_{2} N_{1}\right)}
\end{aligned}
$$

which is the usual form of the $s u(3)$ generating function (cf. section 2.5 of [1]).

\section{A.2. Extension of the determinantal formula methods to fusion rules: the $\widehat{s u}(3)$ case}

The starting point for the derivation of the $\widehat{s u}(3)$ fusion generating function is the generating function for fusions of the form

$$
\left[k-\lambda_{1}-\lambda_{2}, \lambda_{1}, \lambda_{2}\right] \times\left[k-\mu_{1}, \mu_{1}, 0\right]
$$

These fusions are known explicitly and the information on their fusion coefficients can be lifted to the following generating function [26]

$$
\begin{aligned}
& F_{1}\left(d, L_{1}, L_{2}, M_{1}, N_{1}, N_{2}\right)= \\
& \quad \frac{1}{(1-d)\left(1-d L_{1} N_{1}\right)\left(1-d L_{2} N_{2}\right)\left(1-d L_{2} M_{1}\right)\left(1-d M_{1} N_{1}\right)\left(1-d L_{1} M_{1} N_{2}\right)}
\end{aligned}
$$

As explained in the previous subsection, the generating function for products

$$
\left[k-\lambda_{1}-\lambda_{2}, \lambda_{1}, \lambda_{2}\right] \times\left[k-\mu_{1}-\mu_{2}, \mu_{1}, 0\right] \times\left[k-\mu_{2}, \mu_{2}, 0\right]
$$


is given by

$$
\begin{aligned}
F_{2}\left(d, L_{1}, L_{2},\right. & \left.M_{1}, M_{2}, N_{1}, N_{2}\right)= \\
& \stackrel{z}{\Omega} \stackrel{R}{\Omega} F_{1}\left(z^{-1} d, L_{1}, L_{2}, M_{1}, R_{1}^{-1}, R_{2}^{-1}\right) F_{1}\left(z, R_{1}, R_{2}, M_{2}, N_{1}, N_{2}\right) .
\end{aligned}
$$

Here the variable $z$ is introduced in order to keep the level fixed in the composition. By the determinantal formula, the generating function is essentially

$$
F_{3}\left(d, L_{1}, L_{2}, M_{1}, M_{2}, N_{1}, N_{2}\right)=\stackrel{\Omega}{\Omega}_{\geq}^{M_{1}}\left(F_{2}-M_{2} M_{1}^{-1} F_{2}\right)
$$

except that the coefficient of $M_{1}^{\mu_{1}} M_{2}^{\mu_{2}}$ is the multiplicity of $\left(\mu_{1}-\mu_{2}, \mu_{2}\right)$. Thus the final generating function is

$$
F_{4}=\stackrel{\Omega}{\Omega}_{\geq}^{M_{1}} F_{3}\left(d, L_{1}, L_{2}, M_{1}, M_{2} M_{1}^{-1}, N_{1}, N_{2}\right)
$$

This reproduces the generating function given in [4] and re-derived above.

\section{A.3. Determinantal formula methods applied to the $\widehat{s p}(4)$ and $\widehat{s u}(4)$ cases}

In principle, the above procedure can be used to calculate the fusion rule generating functions for $\widehat{s u}(4)$ and $\widehat{s p}(4)$. Unfortunately, the intermediate expressions are too large to be manageable, even when manipulated with computer assistance. However, it is possible to calculate the specialisation of these generating functions with all but one variable, the level-grading variable, set equal to 1 . For example, in the above calculation for $\widehat{s u}(3)$ we could have set $L_{1}=L_{2}=N_{1}=N_{2}=1$ at the start of the calculation since they are not needed at any intermediate steps. Similarly we can set $M_{2}=M_{1}^{-1}$ at the last step which has the effect of setting $M_{2}=1$ in the final generating function. If we set all variables equal to 1, except the one that keeps track of the level, then the resulting generating function $G(d)$ counts the number of independent couplings at each level. The $\widehat{s u}(4)$ and $\widehat{s p}(4)$ specialised generating functions have been calculated in this way and the results are:

$$
G^{\widehat{s u}(4)}(d)=\frac{d^{6}+4 d^{5}+13 d^{4}+16 d^{3}+13 d^{2}+4 d+1}{(1-d)^{12}\left(1-d^{2}\right)}
$$

and

$$
G^{\widehat{s p}(4)}(d)=\frac{d^{4}+2 d^{3}+5 d^{2}+2 d+1}{(1-d)^{9}(1+d)}
$$


These expressions agree with the specialisation of the generating functions found in sections 6 and 7; this thus provides a very strong independent verification of these results. In particular, it corroborates the closure of our set of fusion elementary couplings.

Although we will not present the details of this derivation, we would like to draw attention to some technical issues. There are potentially two problems which could arise in using the determinantal expansions. The first problem is that the determinant may contain terms which have level higher than the initial representation. For example in $\widehat{s u}(3)$ at level 1 the determinantal expansion of the representation $(0,1)$ is

$$
(0,1)=\operatorname{det}\left(\begin{array}{ll}
(1,0) & (0,0) \\
(2,0) & (1,0)
\end{array}\right)
$$

The representation $(2,0)$ is integrable only at level 2 and greater. However it can be shown, using the modification rules of [24], that all such terms in the determinant vanish identically in the $\widehat{s p}(2 n)$ and $\widehat{s u}(n)$ fusion rings. Thus, when computing with the determinantal expansions at a given level, we need only consider terms corresponding to representations which exist at that level.

The second complication which can arise is in a sense the converse of the first. There are representations which occur only at levels strictly greater than $k$, but which have determinantal expansions which contain products which are defined at level $k$. This does not occur for the $s u(n)$ determinants. However for $s p(4)$ this problem can happen. The determinant formula for $s p(4)$ is

$$
\left(\lambda_{1}, \lambda_{2}\right)=\operatorname{det}\left(\begin{array}{cc}
\left(\lambda_{1}+\lambda_{2}, 0\right) & \left(\lambda_{2}-1,0\right) \\
\left(\lambda_{1}+\lambda_{2}+1,0\right)+\left(\lambda_{1}+\lambda_{2}-1,0\right) & \left(\lambda_{2}, 0\right)+\left(\lambda_{2}-2,0\right)
\end{array}\right) .
$$

Take for instance the representation $(0,2)$ which does not exist for level 1 . However the determinant formula yields

$$
(0,2)=(2,0) \otimes(2,0)-(3,0) \otimes(1,0)-(1,0) \otimes(1,0)+(2,0) \otimes(0,0)
$$

The only product which is defined at level 1 is $(1,0) \otimes(1,0)=(0,1)$. Thus the above determinant yields the following modification rule: $(0,2)=-(0,1)$ for $\widehat{s p}(4)$ at level 1 (see [24] for more details). Therefore, before converting the exponent of $M_{1}$ into a Dynkin label, we must ensure that it is less than or equal to the exponent of $d$. This can be achieved by replacing $M_{1}$ by $M_{1} y^{-1}$ and $d$ by $d y$ and then projecting onto non-negative powers of $y$ and finally setting $y=1$. 


\section{A.4. Duality}

As described in [24] and references therein, there is a duality between fusion rules for $\widehat{s u}(n)$ at level $k$ and fusion rules for $\widehat{s u}(k)$ at level $n$. This duality is somewhat involved when using standard Young tableaux. However, it can be clearly seen using contravariant tableaux. This duality can be used to provide a very nice nontrivial check of the $\widehat{s u}(4)$ generating function.

As discussed above, if all the grading variables in the $\widehat{s u}(4)$ fusion generating function are set equal to 1, except for the one associated to the level, we obtain (A.16). However in order to use a duality argument to compare this expression with other generating functions, it needs some modifications. Duality maps Young tableaux to conjugate Young tableaux. For example $\widehat{s u}(3)$ at level 4 has

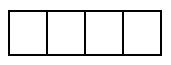

as a possible tableau and this maps to

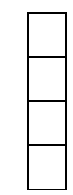

in $\widehat{s u}(4)$ at level 3 . In other words we have to include in the generating functions the terms corresponding to tableaux which have columns of length $n$ in $\widehat{s u}(n)$. If $\mathcal{F}_{n}\left(d, L_{1}, \ldots\right)$ stands for the original $\widehat{s u}(n)$ fusion generating function, then the procedure for incorporating tableaux augmented by columns of length $n$ - while maintaining the first row smaller or equal to $k$ - amounts to calculate

$$
\left.g_{n}(d) \equiv \frac{\partial^{2}}{\partial x \partial y} x y \mathcal{F}_{n}\left(d x y, x^{-1} L_{1}, x^{-1} L_{2}, \ldots, y^{-1} M_{1}, y^{-1} M_{2}, \ldots, N_{1}, N_{2}, \ldots\right)\right|_{x=y=1} .
$$

The effect of this operation is to multiply

$$
d^{k} L_{1}^{\lambda_{1}} L_{2}^{\lambda_{2}} \ldots M_{1}^{\mu_{1}} M_{2}^{\mu_{2}} \ldots N_{1}^{\nu_{1}} N_{2}^{\nu_{2}} \quad \text { by } \quad\left(k-\lambda_{1}-\lambda_{2} \ldots+1\right)\left(k-\mu_{1}-\mu_{2} \ldots+1\right)
$$

which is the factor needed to add in all the Young tableaux with all allowed numbers of columns of length $n$. In other words, the $\widehat{s u}(3)$ tableau $\square$ at level 5 should appear in following equivalent forms:
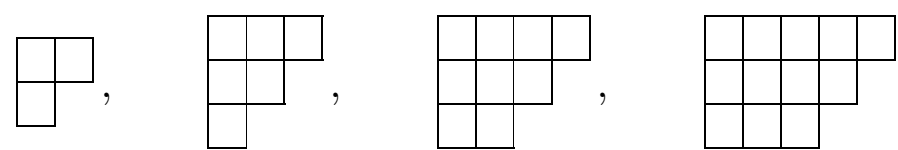
that is, it should be counted four times. Doing this and setting all Dynkin-grading variables equal to 1 leads to the following generating functions:

$$
\begin{aligned}
& g_{0}=\frac{1}{1-d} \quad g_{1}=\frac{1+d}{(1-d)^{3}} \quad g_{2}=\frac{1+3 d+d^{2}}{(1-d)^{6}} \quad g_{3}=\frac{d^{4}+6 d^{3}+10 d^{2}+6 d+1}{(1-d)^{10}} \\
& g_{4}=\frac{d^{10}+13 d^{9}+78 d^{8}+257 d^{7}+513 d^{6}+642 d^{5}+513 d^{4}+257 d^{3}+78 d^{2}+13 d+1}{(1-d)^{12}\left(1-d^{2}\right)^{3}}
\end{aligned}
$$

The first two functions above correspond to the limiting algebras $\widehat{s u}(0)$ and $\widehat{s u}(1)$. For $\widehat{s u}(0)$, there is only the trivial representation and it occurs at any level. Therefore, there is a single coupling at every level and there are no correction factors: $g_{0}(d)=\sum_{k} d^{k}$. The function $g_{1}$ can be constructed by duality. We start with the generating function for $\widehat{s u}(k)$ fusions at level 1. At level 1, we can ignore all relations between the elementary couplings; moreover, we can keep track only of those elementary couplings that occur at level 1: these are the various products involving the fundamental and the scalar representations. The truncated generating function then reads

$$
\frac{1}{(1-d) \prod_{i}\left[\left(1-d L_{i} N_{i}\right)\left(1-d M_{i} N_{i}\right)\right] \prod_{i, j}\left(1-d L_{i} M_{j} N_{i+j}\right)}
$$

where in the last series of term, the summation is defined modulo $k$ with the understanding that $N_{k}=1$. In this function, we replace $d \rightarrow d x y, L_{i} \rightarrow L_{i} / x, M_{i} \rightarrow M_{i} / y$, multiply the result by $x y$, differentiate with respect to $x, y, d$ and set $x=y=L_{i}=M_{i}=N_{i}=1, d=0$ (to keep only the linear term in $d$ ). This gives $(k+1)^{2}$. Hence we have

$$
g_{1}(d)=\sum_{k=1}^{\infty}(k+1)^{2} d^{k}=\frac{1+d}{(1-d)^{3}}
$$

These functions $g_{n}(d)$ display very nice properties:

1 - the factor $(1-d)$ occurs to the power $(n+2)(n+1) / 2$ in the denominator;

2 - the numerator polynomial $p_{n}(d)$ satisfies $p_{n}(1 / d) d^{\operatorname{deg}\left(p_{n}\right)}=p_{n}(d)$;

3- $p_{n}(d)$ has positive coefficients;

4- the difference between the degree of the numerator and denominator is $2 n$.

The mere fact that $g_{4}(d)$ shares the generic properties of the previous $g_{n}$ functions is supporting evidence for the correctness of the $\widehat{s u}(4)$ generating function. 
The Taylor expansions of the $g_{n}(d)$ functions read:

$$
\begin{array}{llllllllllllll}
g_{0}(d)= & 1 & + & d & + & d^{2} & + & d^{3} & + & d^{4} & + & d^{5} & +\cdots \\
g_{1}(d)= & 1 & + & 4 d & + & 9 d^{2} & + & 16 d^{3} & + & 25 d^{4} & + & 36 d^{5} & +\cdots \\
g_{2}(d)= & 1 & + & 9 d & + & 40 d^{2} & + & 125 d^{3} & + & 315 d^{4} & + & 686 d^{5} & +\cdots \\
g_{3}(d)= & 1 & + & 16 d & + & 125 d^{2} & + & 656 d^{3} & + & 2646 d^{4} & + & 8832 d^{5} & +\cdots \\
g_{4}(d)= & 1 & + & 25 d & + & 315 d^{2} & + & 2646 d^{3} & + & 16720 d^{4} & + & 85212 d^{5} & +\cdots
\end{array}
$$

from which duality (i.e. horizontal versus vertical) is completely manifest. (We stress that the 'built-in duality' for obtaining $g_{1}$ concerns only the second row and the second column.) In particular the first 4 terms 1, 25, 315 and 2646 of the $\widehat{s u}(4)$ function match the coefficients of the 5 -th column. This again provides independent evidence for the correctness of the $\widehat{s u}(4)$ fusion generating function out of which the function $g_{4}$ has been constructed. In particular, this is a decisive test of the necessity of the extra elementary coupling $\widehat{F}$ and an evidence for the absence of further additional elementary couplings.

From the above functions $g_{n}(d)$ we can construct the sum

$$
f(r, d)=g_{0}(d)+g_{1}(d) r+g_{2}(d) r^{2}+g_{3}(d) r^{3}+\ldots
$$

where $r$ is the grading variable associated to the rank +1 (i.e., its exponent is the value of $n$ for $\widehat{s u}(n))$. It satisfies $f(r, d)=f(d, r)$ by duality. We speculate that other symmetry properties might be used to provide an explicit formula for $f(r, d)$.

We can illustrate this dual symmetry in a particular example. Consider the function $\tilde{g}_{n}(d)$ that counts the number of couplings of the representation $[k-1,1,0,0, \ldots, 0]$ with anything in $\widehat{s u}(n)$ at level $k$. Since the Young tableau of $(1,0,0, \ldots, 0)$ is invariant under a duality transformation exchanging $k$ and $n$, by summing up the resulting functions multiplied by $r^{n}$, one should produce an expression $\tilde{f}(r, d)$ symmetric in the interchange of $r$ and $d$. The function $\tilde{g}_{n}(d)$ is calculated as follows in terms of the original $\widehat{s u}(n)$ fusion generating function $\mathcal{F}_{n}$ :

$$
\left.\tilde{g}_{n}(d) \equiv \frac{\partial^{2}}{\partial M_{1} \partial x} x \mathcal{F}_{n}\left(d x, x^{-1} L_{1}, x^{-1} L_{2}, \ldots, M_{1}, 1, \ldots, 1,1 \ldots\right)\right|_{x=y=1, M_{1}=0, L_{1}=\ldots=1}
$$

As explained above, the differentiation with respect to $x$ is required in order to take into account all contributing Young diagrams associated to the first representation $(\lambda)$. The second representation being fixed to be $(1,0,0, \ldots, 0)$, does not require an adjusting multiplication factor. Setting the variable $M_{1}=0$, after having differentiated with respect 
to it, simply serves to select the term linear in $M_{1}$. Since the representation $(1,0,0, \ldots, 0)$ does not exist for $\widehat{s u}(0), \tilde{g}_{0}(d)=0$. The function $\tilde{g}_{1}(d)$ is found by duality as explained previously. The first few $\widehat{s u}(n)$ functions $\tilde{g}_{n}(d)$ are found to be:

$$
\begin{array}{ll}
\tilde{g}_{1}(d)=\frac{2 d-d^{2}}{(1-d)^{2}} & \tilde{g}_{2}(d)=\frac{3 d-d^{2}}{(1-d)^{3}} \\
\tilde{g}_{3}(d)=\frac{4 d-d^{2}}{(1-d)^{4}} & \tilde{g}_{4}(d)=\frac{5 d-d^{2}}{(1-d)^{5}}
\end{array}
$$

Fortunately, the general pattern is clear: the expression of $\tilde{g}_{n}$ is easily guessed to be:

$$
\tilde{g}_{n}(d)=\frac{(n+1) d-d^{2}}{(1-d)^{n+1}} \quad n \geq 1
$$

From this exact form of $g_{n}(d)$, we can write down readily the exact expression for the sum

$$
\tilde{f}(r, d)=\sum_{n=1}^{\infty} \tilde{g}_{n}(d) r^{n}=\frac{d r(2-d-r)}{(1-d-r)^{2}}
$$

The result is manifestly invariant under the duality transformation that interchanges $r$ and $d$.

\section{Appendix B. Status of previous conjectures}

In this appendix, we would like to clarify the relation between the present work and our previous ones and state precisely in what sense our previous conjectures are either embodied in the present reformulation of the problem or have been proved.

A general approach to the construction of generating functions for fusion rules was proposed in [4]. It was based on the following two conjectures:

1) Every coupling is characterised by a threshold level $k_{0}$. The multiplicity of a triple product at level $k$ is given by the number of couplings with threshold levels $\leq k$.

2) There is a choice of forbidden couplings such that the threshold level of a coupling is the sum of the threshold levels of its components.

As already mentioned, it can be shown [6] that conjecture 1 is a consequence a sharpened formulation of the depth rule [26]. This leaves us with a single conjecture which we rename: 
Conjecture I: There is a choice of forbidden couplings such that the threshold level of a coupling is obtained from the sum of the threshold levels of the elementary couplings that appear in its decomposition.

In the formulation of conjecture I, the element of 'choice' refers to the fact that both sides of a tensor-product relation do not always have the same threshold level and which one is taken as the forbidden coupling makes a difference in the generating function for fusion rules. With the notion of a set of elementary fusion couplings, which includes the scalar one (this is a new feature of the present work), all relations acquire equal threshold levels and this choice becomes immaterial. This suggests the following modification of conjecture I:

Conjecture I': The threshold level of a fusion coupling is read off from its decomposition into the elementary fusion couplings.

A interesting aspect of this reformulation of the conjecture is that it embodies an observation that was presented as a conjecture in [7], namely that the level is always minimised. More precisely, in the choice of forbidden couplings, we should always forbid the one with higher threshold level. This 'minimal level' prescription is automatically taken into consideration here since the relations have identical levels. If one of the products appears in the relation with a factor $\widehat{E}_{0}$, it means that the product without this $\widehat{E}_{0}$ factor occurs at a lower level and it is not forbidden. For instance, the relation $\widehat{E}_{1} \widehat{E}_{3} \widehat{E}_{5}=$ $\widehat{E}_{0} \widehat{E}_{7} \widehat{E}_{8}$ indicates that the coupling $\widehat{E}_{7} \widehat{E}_{8}$ appears at level 2. In the tensor-product relation $E_{1} E_{3} E_{5}=E_{7} E_{8}$, we have thus effectively forbid the higher-level term of the relation.

Once the notion of fusion elementary couplings in terms of which every coupling can be decomposed (conjecture I') is introduced, this naturally calls for a reinterpretation in terms of a fusion basis. It is indeed plain that our conjecture (and the mere existence of threshold level) boils down the fundamental conjecture presented in the text, that is, the existence of a fusion basis. 


\section{REFERENCES}

1. L. Bégin, C. Cummins and P. Mathieu, Generating functions for tensor products,

hep-th/9811113.

2. P. Di Francesco, P. Mathieu, D. Sénéchal, Conformal Field Theory, Springer Verlag 1997.

3. E. Verlinde, Nucl. Phys. B 300 (1988) 389.

4. C.J. Cummins, P. Mathieu and M.A. Walton, Phys. Lett. B254 (1991) 390.

5. A.D. Berenstein and A.Z. Zelevinsky, J. Algebraic Combinat. 1 (1992) 7.

6. A.N. Kirillov, P. Mathieu, D. Sénéchal and M. Walton, Nucl. Phys. B391 (1993) 651.

7. L. Bégin, A.N. Kirillov, P. Mathieu and M. Walton, Lett. Math. Phys. 28 (1993) 257.

8. V.G.Knizhnik and A.B Zamolodchikov, Nucl. Phys. B247 (1984) 83.

9. D.Gepner and E.Witten, Nucl. Phys. B278 (1986) 493.

10. M.A.Walton, Nucl. Phys. B340 (1990) 777; Phys. Lett. B241 (1990) 365.

11. V.G.Kac, Infinite dimensional Lie algebras, 3rd edition, Cambridge Univ. Press. (1990), exercise 13.35 .

12. P.Furlan, A.Ganchev and V.B. Petkova, Nucl. Phys. B343 (1990) 205; J.Fuchs and P.van Driel, Nucl.Phys. B346 (1990) 632.

13. V. Kac and M. Wakimoto, Adv. Ser. Math. Phys. 7 (World Scientific, 1988) 138

14. P. Mathieu and M.A. Walton, Nucl. Phys. B 553 (1999) 533-558.

15. M. Walton, Can. J. Phys. 72 (1994) 527.

16. R.P. Stanley, Duke Math. J. 40 (1973) 607; Combinatorics and Commutative Algebra, (Boston: Birkhauser) (1983).

17. A. Schrijver, Theory of linear and integer programming, Wiley 1986.

18. D. Beklemichev, Cours de géométrie analytique et d'algèbre linéaire, Mir. p. 519.

19. L. Bégin, P. Mathieu and M.A. Walton, Mod. Phys. Lett. A, Vol. 7 (1992) 3255.

20. A.D. Berenstein and A.V. Zelevinsky, J. Geom. Phys. 5 (1989) 453.

21. L. Bégin, P. Mathieu and M.A. Walton, J. Phys. A: Math. Gen. 25 (1992) 135.

22. R.T Sharp and D. Lee, Revista Mexicana de Fisica 20(1971) 203.

23. M.Couture, C.J.Cummins and R.T.Sharp, J.Phys A23 (1990) 1929.

24. C.J. Cummins, J. Phys. A 24 (1991) 391.

25. J. Fuchs, Fortschr. Phys. 42 (1994) 1. 
26. F. Goodman and H. Wenzl, Adv. Math. 82 (1990) 244. 